hep-th/0312022

HUTP-03/A078

IHES $/ \mathrm{P} / 03 / 65$

ITEP-TH-60/03

\title{
Quantum Foam and Topological Strings
}

\author{
Amer Iqbal $^{a}$, Nikita Nekrasov ${ }^{b \dagger}$, Andrei Okounkov $^{c}$ and Cumrun Vafa ${ }^{a}$ \\ ${ }^{a}$ Jefferson Physical Laboratory, Harvard University \\ Cambridge, MA 02138, USA \\ ${ }^{b}$ Institut des Hautes Etudes Scientifiques \\ Bures-sur-Yvette, F-91440, France \\ ${ }^{c}$ Department of Mathematics, Princeton University \\ Princeton, NJ 08544, USA
}

\begin{abstract}
We find an interpretation of the recent connection found between topological strings on Calabi-Yau threefolds and crystal melting: Summing over statistical mechanical configuration of melting crystal is equivalent to a quantum gravitational path integral involving fluctuations of Kähler geometry and topology. We show how the limit shape of the melting crystal emerges as the average geometry and topology of the quantum foam at the string scale. The geometry is classical at large length scales, modified to a smooth limit shape dictated by mirror geometry at string scale and is a quantum foam at area scales $\sim g_{s} \alpha^{\prime}$.
\end{abstract}

December 2003

$\dagger$ On leave of absence from: ITEP, Moscow, 117259, Russia 


\section{Introduction}

The idea that quantum gravity should lead to wild fluctuations of topology and geometry at the Planck scale is an old idea [1, 2]. Despite the plausible intuitive nature of this idea it has not become a precise part of our current understanding of quantum gravity. Even with the advent of superstring theory as a prime candidate for a theory of quantum gravity, we still have a long way to go to understand the geometry of space at short distances. For example for type IIA superstrings in $\mathbf{R}^{10}$, even for small $g_{s}$, we do not know how the spacetime looks like at the Planck scale $l_{p}=g_{s}^{1 / 4} l_{s}$. Trying to probe geometries at such a distance scale requires high center of mass energies which lead to creation of black holes and the puzzles associated with it.

A good laboratory for studying these questions is the topological strings. Topological A-model strings [3] involves from the worldsheet viewpoint sums over holomorphic maps to the target space. The critical case corresponds to the target space being Calabi-Yau threefolds. From the target space perspective one is considering a gravity theory known as 'Kähler gravity' [4]. Roughly speaking one is integrating over the space of all Kähler metrics on the Calabi-Yau manifold. Of course, the action of this gravity theory is not just the Einstein action, as it would be a total derivative on the Kähler manifold.

Topological string is rich enough to have many features familiar from superstrings: Lagrangian D-branes, (known as A-branes), large $N$ open string/closed string dualities [5] etc. Moreover, in the Berkovits formalism, topological string amplitudes can be viewed as part of the worldsheet theory of superstring (for a recent discussion of this see [6]), where they are interpreted as F-term amplitudes [7, [8]. It is thus interesting to ask whether or not we can have a more clear picture of the gravitational quantum foam in this theory.

Recently a duality was discovered in [9] which related topological string amplitudes in the A-model to a statistical mechanical partition function involving crystal melting. Such a duality between a quantum theory and a classical system has one well known analog: Quantum field theories can typically be viewed as sums over classical configurations of fields with some weights determined by the action. It is thus natural to try to interpret the configurations of melting crystal as target space field theory of the A-model, which should be a gravitational theory involving fluctuations of topology and geometry. Thus the partition function of the crystal melting would get mapped to a weighted sum over various topologies. 


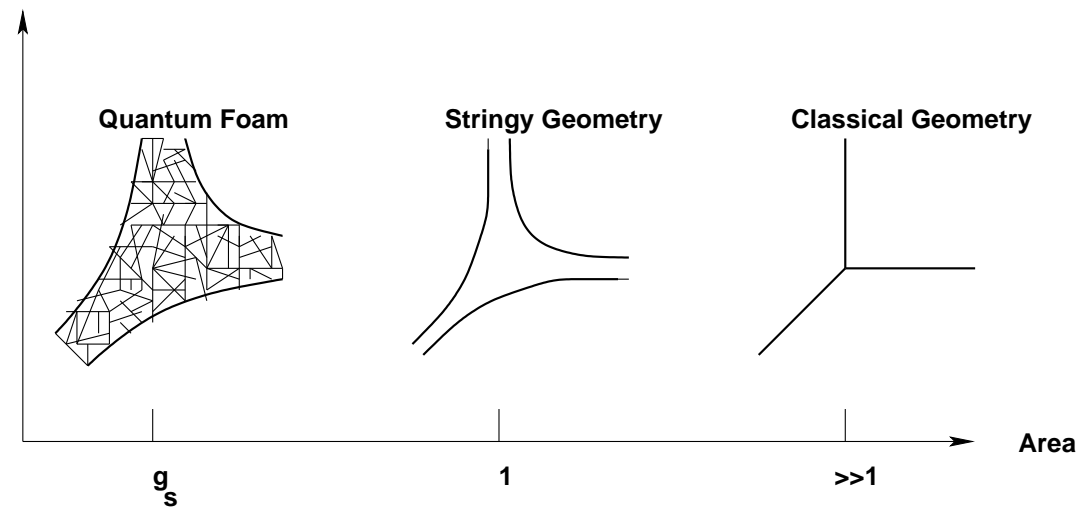

Fig. 1: The diagram shows how the geometry varies as we change the area scale, drawn in string units.

In this paper we find that this is indeed a consistent interpretation of this duality: We find that at short distance when the area is of order of $g_{s} l_{s}^{2}$ where $l_{s}=\sqrt{\alpha}^{\prime}$ is the string scale, (assuming $g_{s}<<1$ ) a gravitational quantum foam takes over. The foam gets identified with the fluctuating boundary configuration of the crystal. The geometry and topology fluctuate wildly at this scale. At larger scales, at the string scale, a smooth geometry takes over which is the average fluctuation of the foam dictated by geometry of the mirror manifold. This is a macroscopic regime for the crystal where a smooth limit shape emerges for the boundary of the molten crystal. At yet larger scales the classical geometry description takes over. At these scales, the crystal appears not to have molten at all. See fig. 1. It is also natural to see how this picture can be embedded in superstrings. One possible approach to this idea is currently under investigation [10].

What we find is that the quantum fluctuations of topology involves blowing up the original Calabi-Yau manifold $X$ along points and holomorphic curves. The blown up space $\widehat{X}$ is no longer Calabi-Yau. It comes canonically with a line bundle $L$ whose local holomorphic sections are identified with the local holomorphic functions on $X$, which vanish along the curves and points we have blown up. The new Kähler form on $\widehat{X}$ is obtained from the original one on $X$ up to the addition of the first Chern class of $L$ times $g_{s}$. Precise definition of summing over "quantum Kähler structures" consists of integrating over "ideal sheaves", which are "torsion free sheaves with vanishing first Chern class". In string theory we often deal with these objects when considering gauge fields supported on D-branes. More physically, we replace the integration over Kähler geometries and topologies by the path-integral of a $U(1)$ gauge theory on $X$ (related to a topological twisting of maximally 
supersymmetric gauge theory). Thus our proposal is similar in spirit to $S L_{2}(\mathbf{R})$ and $S L_{2}(\mathbf{C})$ gauge field interpretations of two and three dimensional gravities respectively.

The ideal sheaf is the generalization of the line bundle, and the moduli space of ideal sheaves is the generalization of the moduli space of instanton solutions in the 6 dimensional gauge theory on $X$. The torsion free sheaves fail to be a line bundle in real codimension four. The Calabi-Yau $X$ can be blown up along this locus to produce another space $\widehat{X}$. Torsion free sheaves can be lifted to $\widehat{X}$ where they become line bundles. There is no one to one correspondence between the sheaves on $X$ and blown up geometries $\widehat{X}$, and in some sense there are more sheaves than geometries. We interpret this mismatch as a lesson of quantum string theory for quantum gravity: The path-integral space for quantum gravity should include classical topologies and geometries. However the actual space we integrate over may well be bigger than that given strictly by manifolds with arbitrary topology and metric, as happens for topological strings. Thus we conclude, that for topological strings a gauge theory is the fundamental description of gravity at all scales including the Planck scale, where it leads to a quantum gravitational foam. A related example of this, as we will note later, is that of $3 \mathrm{~d}$ gravity theory and its formulation in terms of $S L(2, \mathbf{C})$ ChernSimons theory [11].

The plan of this paper is as follows: In section 2 we present the basic idea. In section 3 we discuss aspects of toric geometry which is relevant for the main class of non-compact examples studied in this paper. It is also necessary for understanding the nature of the quantum foam. In section 4 we apply this to the $\mathbf{C}^{3}$ case. In section 5 we discuss the generalization of this to the case of toric 3 -folds. Some of the more technical discussions are presented in section 6 . In particular in that section we propose more precisely what Kähler gravity is in terms of the $U(1)$ gauge theory of a maximally supersymmetric, topologically twisted theory. We also show how the idea of localization for toric geometries works in this context by considering certain deformations, similar to the approach taken for $4 \mathrm{~d}$ instantons [12]. In section 7 we briefly discuss generalizations to the compact case.

\section{The Basic Idea}

Topological A-model involves, from the worldsheet perspective summing over holomorphic maps to the target [3]. The critical case, which is the case of interest in this paper, is when the target space is a Calabi-Yau threefold, which we denote by $X$. The (complexified) Kähler class of the target space, represented by a closed $(1,1)$ form $k$, plays 
a key role: It weights worldsheet instantons by $e^{-k_{\Sigma}}$ where $k_{\Sigma}$ denotes the integral of $k$ over the image of the worldsheet $\Sigma$.

As far as the target geometry is concerned at the level of genus 0 worldsheet, i.e., string theory tree level, one is studying quantum intersection theory. This means, roughly speaking, one is asking whether cycles intersect, up to a fuzziness involving holomorphic spheres. Thus the target geometry is inherently 'fuzzy' at the string scale.

The target space description of the field theory, should involve integration over the space of all Kähler metrics on the Calabi-Yau, with a fixed complex structure. This was studied in [4] following earlier works [13,7], where it was found that one is studying 'Kähler gravity'. Part of the difficulty in implementing [4] as a practical method for computing topological A-model amplitudes is that one expects that a 'stringy' geometry should take over the classical description of the geometry at distances of order of string scale, where the worldsheet instantons will not be suppressed. Thus the lack of a precise meaning for what could one mean by a 'stringy Kähler gravity' is an obstacle to this program. At any rate, it was found that the classical solutions of the theory is given by Kähler forms $k$, and the action evaluated at these points is given by

$$
\mathcal{S}=\frac{1}{g_{s}^{2}} \int_{X} \frac{1}{3 !} k \wedge k \wedge k
$$

On the other hand recent results indicate that the Kähler form in topological strings is quantized. The first hint of that emerged in [5] where it was found that topological string on resolved conifold is equivalent to $U(N)$ Chern-Simons on $S^{3}$ provided that the Kähler class of the blown up $\mathbf{P}^{\mathbf{1}}$ is identified with $k\left(\mathbf{P}^{1}\right)=N g_{s}$. More recently [14] it was found that quite generically if one considers $N$ Lagrangian D-branes (known as A-branes) inside a CY, then the integral of $k$ along 2-cycles surrounding them jumps by $N g_{s}$, generalizing the observation in [5]. It is thus natural to suspect that quite generally $k$ should be viewed as quantized in units of $g_{s}$ (for quantization of areas in a different context see e.g., [15]). In other words we have

$$
k=F g_{s},
$$

where $F$, being an integral $(1,1)$ form, is the curvature of a $U(1)$ bundle over the CalabiYau. 
Note that $\int_{X} k^{3} / g_{s}^{3}$ could be viewed as the index of the $\bar{\partial}$ operator coupled to a gauge field with field strength $k / g_{s}$ l. Thus we can view $\mathcal{S}$ as counting the net number of holomorphic sections of the bundle, up to a factor of $g_{s}$, i.e.

$$
Z=\sum e^{\mathcal{S}}=\sum \exp \left(N g_{s}\right)
$$

where $N$ is the number (or more precisely the index) of holomorphic sections of this bundle and we sum, in a first approximation, over all line bundles with connection. $N$ can also be viewed as the number of states in the Hilbert space, if we identify

$$
\begin{aligned}
\text { Calabi }- \text { Yau } & \longleftrightarrow \text { phase space } \\
k & \longleftrightarrow \text { symplectic form } \\
g_{s} & \longleftrightarrow \hbar
\end{aligned}
$$

In this context the geometric quantization identifies the states in the Hilbert space with holomorphic sections of the bundle whose curvature is $k / g_{s}$.

It is also interesting to ask whether this Hilbert space has a natural interpretation directly in topological strings. Note that viewing Lagrangian A-branes as magnetic objects, which give rise to $k$ flux, the electric objects which couple minimally to the connection whose curvature is $k$, are 1-dimensional Euclidean objects (0-branes in the more conventional terminology of superstrings). Namely if the 1-dimensional branes bound a disk $D$, their action is weighted by

$$
\exp \left(-\frac{i}{g_{s}} \int_{D} k\right)
$$

That this is well defined independently of the choice of the bounding disk $D$, follows because $k$ is quantized in units of $g_{s}$. These branes can also be viewed as induced by turning on a $U(1)$ field strength inside a Lagrangian A-brane. Thus the partition function of topological A-model seems to be counting the number of such states for each configuration of Kähler moduli.

It is also useful to expand $k$ near a fixed background geometry $k_{0}$, as often is needed in topological strings. In this context it is natural to write the fluctuations in terms of the field strength of a $U(1)$ bundle. It is also natural to fix the Kähler class of the macroscopic geometry; in other words we write

$$
k=k_{0}+g_{s} F
$$

1 We neglect the gravitational corrections to the index for the moment 
and require that the fluctuation has no periods along 2-cycles of the macroscopic Kähler geometry i.e., $\int_{\beta} F=0$ for $\beta \in H_{2}(X, \mathbf{Z})$. This leads to

$$
\mathcal{S}=\frac{1}{g_{s}^{2}} \frac{1}{3 !} \int_{X} k_{0}^{3}+\frac{1}{2} \int_{X} F \wedge F \wedge k_{0}+g_{s} \int_{X} \frac{1}{3 !} F \wedge F \wedge F
$$

Thus, apart from the constant background piece $k_{0}^{3}$, this can be written as

$$
Z=\sum \exp (\mathcal{S})=\sum q^{c h_{3}} \prod_{i} Q_{i}^{\int_{C \vee} c h_{2}}
$$

where

$$
q=e^{g_{s}} \quad Q_{i}=e^{-\int_{C_{i}} k_{0}}
$$

where $C_{i}, C_{i}^{\vee}$ are the dual bases of $H_{2}(X, \mathbf{Z})$ and $H_{4}(X, \mathbf{Z})$, and $c h_{3}$ and $c h_{2}$ denote the corresponding Chern classes of the $U(1)$ bundle over the Calabi-Yau 3-fold. Note that the condition on $F$ above means that it realizes trivial cohomology class. How come the second and the third Chern characters do not vanish? The resolution of the puzzle is that $F$ corresponds to the singular $U(1)$ gauge field on $X$. To make it nonsingular one either blows up the space $X$ or relaxes the notion of the line bundle. In fact, the latter approach is canonically related to the former, as we shall explain in more detail in the sections 3 and 7. The natural replacement for the holomorphic line bundle $\mathcal{L}$ is the rank one torsion free sheaf $\mathcal{I}$ with the same first Chern class.2

2 There is exact sequence of sheaves:

$$
0 \longrightarrow \mathcal{I} \longrightarrow \mathcal{L} \longrightarrow S_{Z} \longrightarrow 0
$$

where $S_{Z}=i_{*} \mathcal{O}_{Z}$ is the sheaf supported on the submanifold $Z$ of real codimension four, which is the union of curves and points. From (2.4) one derives, e.g. for $Z$, which is a union of curves $\Sigma_{\alpha}$ and points $P_{a}$ :

$$
\operatorname{ch}(\mathcal{I})=\operatorname{ch}(\mathcal{L})-\operatorname{ch} S_{Z}
$$

and in particular:

$$
\begin{gathered}
\operatorname{ch}_{2}(\mathcal{I})=\frac{1}{2} c_{1}(\mathcal{L})^{2}-\sum_{\alpha} P . D .\left[\Sigma_{\alpha}\right] \\
\operatorname{ch}_{3}(\mathcal{I})=\frac{1}{6} c_{1}(\mathcal{L})^{3}-\sum_{a} P . D .\left[P_{a}\right]+\sum_{\alpha} c_{1}\left(\Sigma_{\alpha}\right) P . D .\left[\Sigma_{\alpha}\right]
\end{gathered}
$$

(we used Calabi-Yau condition). It is these correction terms, supported on $Z$ which make our formula (2.2) nontrivial. 
From quantum gravity point of view the sum in (2.3) should be over a suitable class of geometries with a suitable measure. What we will do in this paper is to propose a precise definition of this sum in terms of a topologically twisted maximally supersymmetric $U(1)$ gauge theory on the Calabi-Yau. We find that for toric cases this localizes to a very simple formula, leading to the rules for the statistical mechanics of crystal melting. It is not a priori clear why a simple formula such as this should have worked in an exact way, as we shall find in this paper.

More precisely what we will find is that if we study toric Calabi-Yau, the above sum could be localized to toric geometries, with quantized Kähler moduli. From this point of view we can describe this as a singular Kähler moduli on the original space. This is somewhat analogous to instantons on non-commutative $\mathbf{C}^{2}$ which can also be viewed, in some cases, as smooth instantons on certain blowup of $\mathbf{C}^{2}$ [16]. In fact the sum we perform can also be viewed as summing over 3-dimensional analogs of instantons on noncommutative space. However it is more natural for our case to view this as a Kähler moduli on a smooth blown up geometry. The main case we will concentrate on is for that of toric Calabi-Yau 3-folds. In fact the sum we do is an enlargement of the space of all quantized Kähler moduli on toric geometries. This is also somewhat analogous to the $3 \mathrm{~d}$ gravity described in terms of $S L(2, \mathbf{C})$ Chern-Simons connection where the relevant space of connections is an enlargement of the space of allowed metrics [11] (see [17] for a recent discussion of this theory). For non-toric geometries we shall also formulate a precise meaning to the above sum, in terms of mathematical objects known as torsion free sheaves, which are nothing but the ideal sheaves in our case. This involves the notion of what singular $U(1)$ 'instanton configurations' mean for 3-folds.

We will develop these ideas further, after we discuss some preliminaries of toric geometries in the next section. In section 4 and 5 we assume a simple localization holds and give an interpretation of the result of [9]. Physically the localization should be viewed, as we will note below, as the geometry seen by brane probes which are themselves toric. In section 6 we define more precisely what the gauge theory is and why it localizes.

\section{Toric Geometry Preliminaries}

It is crucial for this paper to have a simple intuitive grasp of toric geometries. To this end here we present a self-contained introduction to certain aspects of toric geometries relevant for this paper. 
Let us start with the 1-dimensional complex plane $\mathbf{C}$. Let us denote its coordinate by $z$. There is a $U(1)$ action which corresponds to phase multiplication. We can view $\mathbf{C}$ as

$$
z=|z| e^{i \theta}
$$

The standard Kähler form on $\mathbf{C}$ can be viewed as

$$
k=i d z \wedge d \bar{z}=d \theta \wedge d|z|^{2}
$$

Let us define

$$
p=|z|^{2}
$$

the moment map, in terms of which the Kähler form is given by

$$
k=d \theta \wedge d p
$$

We can view $\mathbf{C}$ as a circle, parameterized by $\theta$, fibered over positive half-line $p \geq 0$.

Let us view $\mathbf{C}$ as the phase space with $k$ as the symplectic form. Furthermore suppose we were to write a basis for the Hilbert space for the quantization of this phase space. Motivated by our discussion of the previous section, we introduce the Planck constant $\hbar=g_{s}$ and view $\theta$ as the space coordinate,

$$
[\theta, p]=i g_{s}
$$

A basis for the Hilbert space can be taken to be

$$
\psi_{n}(\theta)=\exp (i n \theta),
$$

which leads to

$$
p=n g_{s} .
$$




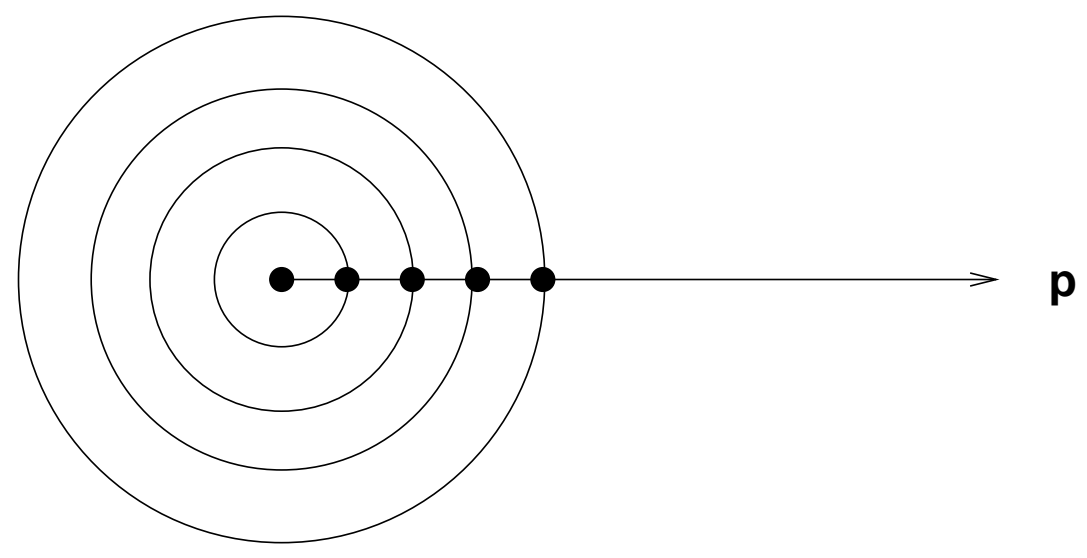

Fig. 2: The diagram shows how the $p=|z|^{2}$ half-line can be chosen as the Lagrangian subspace of complex plane. The states of the Hilbert space are identified with $p=n g_{s}$ for positive integer $n$.

Here $n$ should be integer, because of the periodicity of $\theta$ and $n \geq 0$, by the fact that the momentum $p \geq 0$. We can identify the space of states as integer lattice points in the positive half-line parameterized by $p$, separated by lattice spacing $g_{s}$. The above result of an integer spectrum for $p=|z|^{2}$ is familiar in the context of one dimensional Harmonic oscillator, with the identification of $H=p=|z|^{2}$.

We can also get the same set of states by geometric quantization. What this means is that we view $k$ as the curvature of a $U(1)$ bundle, up to a factor of $\hbar=g_{s}$

$$
k=g_{s} F
$$

and we are looking for holomorphic sections of the bundle given by

$$
\bar{D}_{A} \psi=(\bar{\partial}+\bar{A}) \psi=0
$$

In this case $\bar{A}=z$ and so a basis for the solutions are given by

$$
\psi_{n}=z^{n} e^{-z \bar{z}}
$$

Note that the wave function $e^{i n \theta}$ corresponds to the phase in the $z^{n}$ factor above. Both of these viewpoints will be useful for us.

Note that holomorphic functions on $\mathbf{C}$ act on the Hilbert space. This is generated by functions of $z$ and we have

$$
z \psi_{n}=\psi_{n+1}
$$


This is a general fact about geometric quantization:

$$
\left[z, \bar{D}_{A}\right]=0
$$

and thus holomorphic functions are realized as natural operators on the Hilbert space.

The generalization of this to $n$-dimensional complex plane is straight-forward. The space of states in this case can be identified with an $n$-dimensional crystal sitting at positive integral points of $\left(p_{1}, \ldots, p_{n}\right)$ separated by $g_{s}$. In the case of $n=3$ this gives a three-dimensional cubic crystal, filling the positive octant (which can also be identified with the states of a three dimensional harmonic oscillator).

So far we have considered the simplest toric geometries. For more interesting ones we add more coordinates and impose identifications in the holomorphic terminology, or constraints modulo gauge equivalences in the linear sigma model terminology [18]. In general we will be considering $n+r$ variables $z_{i}$, where $i=1, \ldots, n+r$, with $r$ identifications/constraints. The identifications/constraints are encoded in terms of $r$ sets of integral charge vectors $Q_{i}^{a}$ where $a=1, \ldots, r$ and $i=1, \ldots, n+r$. In the holomorphic description we identify

$$
z_{i} \sim \lambda_{a}^{Q_{i}^{a}} z_{i}
$$

for each $a$, where $\lambda_{a} \in \mathbf{C}^{*}$ and certain loci are deleted. In the linear sigma model set up we consider the $r$ constraints

$$
\sum_{i} Q_{i}^{a}\left|z_{i}\right|^{2}=\sum_{i} Q_{i}^{a} p_{i}=t^{a}
$$

modulo $U(1)^{r}$ gauge symmetry given by

$$
z_{i} \rightarrow e^{i \theta^{a} Q_{i}^{a}} z_{i}
$$

The above constraints (3.1) are linear in the $p_{i}$ variables. The space $\Delta(X)$ of $p_{i}$ consistent with the above equations is generically $n$ dimensional subspace of $\mathbf{R}_{\geq 0}^{n}$. It is also easy to see that this subspace is convex: If $p_{i}^{1}>0$ and $p_{i}^{2}>0$ satisfy the charge constraints, then $\lambda p_{i}^{1}+(1-\lambda) p_{i}^{2}$ are positive and also satisfy the constraints for $1 \geq \lambda \geq 0$. The space $\Delta(X)$ is the quotient $X$ by the torus $\mathbf{T}^{n}$, which is in turn the quotient $\mathbf{T}^{r+n} / \mathbf{T}^{r}$.

In the above $t^{a}$ denote the various Kähler moduli of the toric geometry. We would also like to consider these spaces as phase spaces and do quantum mechanics on them. In doing so we find that for there to be a well defined wave functions we need the Kähler 


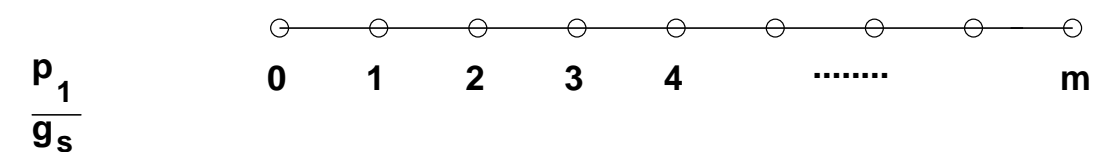

Fig. 3: The toric diagram for $\mathbf{P}^{1}$ is an interval. The sphere arises by recalling that over each point there is a circle which shrinks at the two ends. The diagram shows how the states in the quantum mechanical problem corresponds to integer point in $g_{s}$ units.

moduli $t^{a}$ to be quantized in units of $g_{s}$. This is because if we consider the wave functions as a function of periodic spatial coordinates $\theta_{i}$, single valuedness requires

$$
\psi\left(\theta_{i}\right)=\exp \left(i N_{i} \theta_{i}\right)
$$

where $N_{i}$ are integers. However $N_{i}$ cannot be arbitrary, because the corresponding momenta $p_{i}=N_{i} g_{s}$ should satisfy (3.1):

$$
\sum_{i} Q_{i}^{a} N_{i} g_{s}=t^{a}
$$

For this to have solutions, we need $t^{a}$ to be quantized in units of $g_{s}$ :

$$
t^{a}=m^{a} g_{s}
$$

which leads to

$$
\sum_{i} Q_{i}^{a} N_{i}=m^{a}
$$

The set of points $\left\{N_{i}\right\} \subset \mathbf{Z}_{\geq 0}^{n+r}$ satisfying (3.3) label the vector space which span the Hilbert space $\mathcal{H}$ corresponding to quantization of the space. They could also be viewed as the space of sections of the holomorphic bundle with curvature $k=F g_{s}$, in case it is sufficiently positive.

\subsection{Examples}

In order to illustrate these ideas we will construct a few examples.

$\mathbf{P}^{1}$ : First consider a compact example of dimension one, namely $\mathbf{P}^{1}$. This can be realized by two variables $\left(z_{1}, z_{2}\right)$ with one charge vector $(1,1)$. Let $t$ denote the Kähler class. Then we have $p_{1}+p_{2}=t$ 
If we take $t$ to be quantized in $g_{s}$ units, $t=m g_{s}$ then we can identify the states of the Hilbert space with integer points $N_{1} \geq 0, N_{2} \geq 0$, subject to

$$
N_{1}+N_{2}=m .
$$

Note that $N_{2}=m-N_{1}>0$ implies that we can identify this space with lattice points on the positive line satisfying

$$
0 \leq N_{1} \leq m
$$

In particular this space has $m+1$ points, see fig. 3 . The corresponding Kähler class $k$ is identifiable with $g_{s}$ times the curvature corresponding to the line bundle $O(m)$ over $\mathbf{P}^{1}$. This is the bundle whose holomorphic sections can be identified with homogenous degree $m$ polynomials in $z_{1}, z_{2}$. A solution of (3.4),$\left(N_{1}, N_{2}\right)$, corresponds to a monomial $z_{1}^{N_{1}} z_{2}^{N_{2}}$. Thus degree $m$ monomials are naturally identifiable with the above $m+1$ points.

$\mathbf{C}^{2}$ : As our next example we consider a non-compact 2-dimensional space. This will correspond to blowing up $\mathbf{C}^{2}$ at a point. This means we take a point on $\mathbf{C}^{2}$ and replace it with a $\mathbf{P}^{1}$. The geometry near this $\mathbf{P}^{1}$ is $\mathcal{O}(-1) \rightarrow \mathbf{P}^{1}$, and in particular it is not a Calabi-Yau space. We take three variables and take one charge

$$
Q=(1,1,-1) .
$$

Then the momenta satisfy

$$
p_{1}+p_{2}-p_{3}=t .
$$

The $\mathbf{P}^{1}$ is identified with the subspace above with $p_{3}=0$, and the normal bundle $\mathcal{O}(-1)$ over $\mathbf{P}^{1}$ is identified with varying $p_{3} \geq 0$. Again if we quantize $t$ we get for the allowed integral momenta the condition that $N_{i} \geq 0$ and

$$
N_{1}+N_{2}-N_{3}=m .
$$

Note that this can be identified with the space of positive integral points in the quadrant $\left(N_{1}, N_{2}\right)$ with the further restriction that

$$
N_{3}=N_{1}+N_{2}-m \geq 0 .
$$




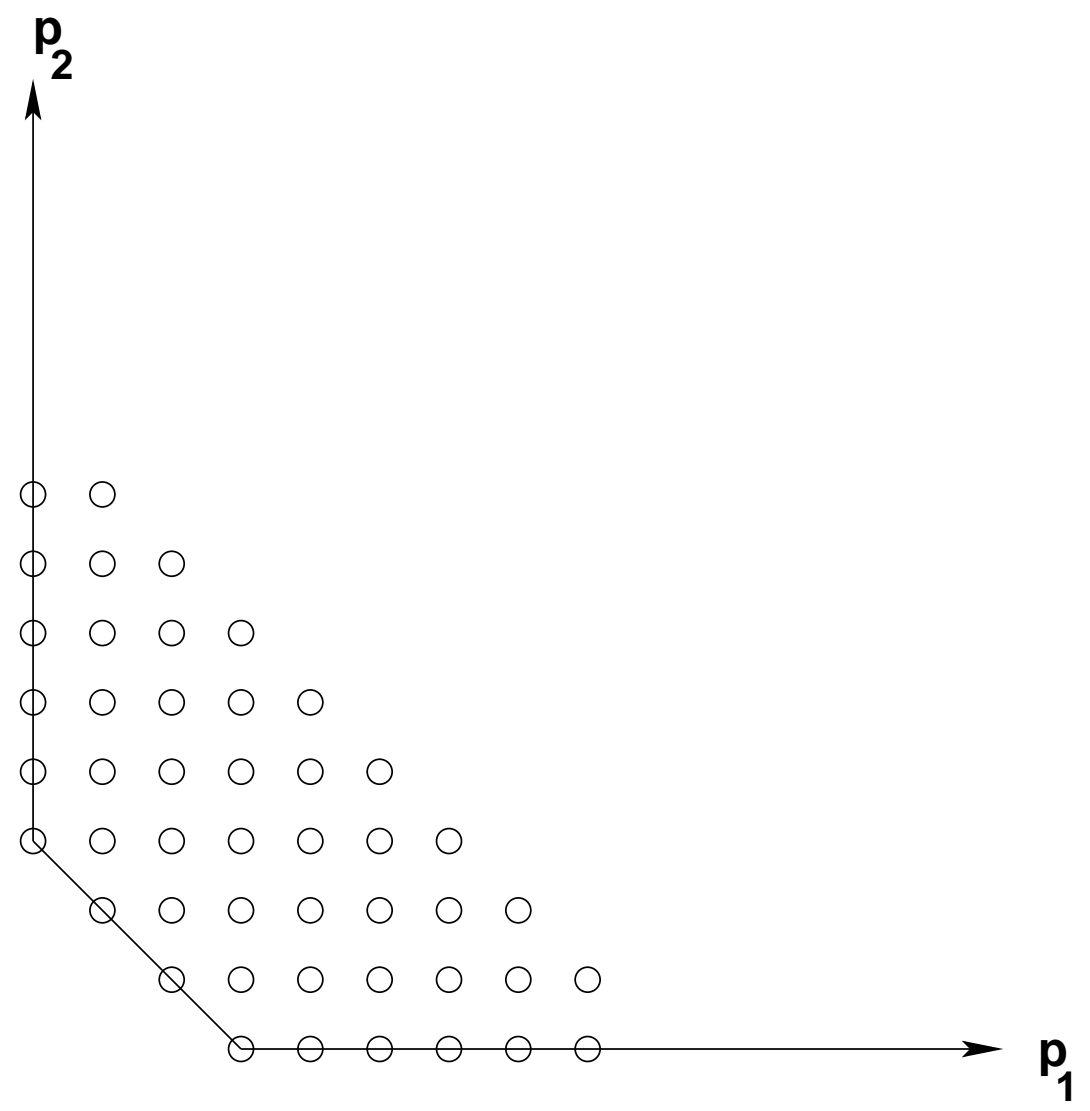

Fig. 4: The diagram shows blowup of a point of $\mathbf{C}^{2}$ and replacing it with a $\mathbf{P}^{1}$. The case depicted in the figure has Kähler class $t=3 g_{s}$.

The Kähler class over $\mathbf{P}^{1}$ corresponds to the $\mathcal{O}(m)$ bundle, as discussed before. This is called blowing up a point in $\mathbf{C}^{2}$ because we have removed the origin and replaced it by a $\mathbf{P}^{1}$, which is represented by an interval in the plane. Note that compared to $\mathbf{C}^{2}$ we have deleted a triangle of points from the space, by blowing up the point at the origin. In other words we have $m(m+1) / 2$ less elements in the Hilbert space (with a natural definition of 'counting' the states of the Hilbert space).

One can also blow up to a singular space. For example let us consider a singular blow up of $\mathbf{C}^{2}$ : consider three variables with one charge

$$
Q=(2,1,-1)
$$

Then we have

$$
2 p_{1}+p_{2}-p_{3}=t
$$


The corresponding space has a $\mathbf{Z}_{2}$ singularity at $p_{1}=t / 2, p_{2}=0, p_{3}=0$. To see this note that the $\mathbf{C}^{*}$ action generated by $Q$ is gauge fixed by going to fixed value of $z_{1}$. But this leaves an extra $Z_{2}$ part of the action which sends $\left(z_{2}, z_{3}\right) \rightarrow\left(-z_{2},-z_{3}\right)$. Note again in this example if we wish an integral subspace of states we can take $t=m g_{s}$ leading to the positive integral momenta constrained by

$$
2 N_{1}+N_{2}-N_{3}=m .
$$

We can also blow up more than one point on $\mathbf{C}^{2}$. For example consider four variable with two charges

$$
\begin{aligned}
& Q^{1}=(1,1,-1,0), \\
& Q^{2}=(1,0,1,-1) .
\end{aligned}
$$

Giving

$$
\begin{aligned}
& p_{1}+p_{2}-p_{3}=t^{1}, \\
& p_{1}+p_{3}-p_{4}=t^{2} .
\end{aligned}
$$

This space corresponds to the subspace of the positive $\left(p_{1}, p_{2}\right)$ quadrant satisfying

$$
\begin{gathered}
p_{1}+p_{2} \geq t^{1}, \\
2 p_{1}+p_{2} \geq t^{1}+t^{2} .
\end{gathered}
$$

Again, if we wish to obtain integral points we can consider quantizing $t^{1}, t^{2}$ in units of $g_{s}$ leading to

$$
\begin{gathered}
N_{1}+N_{2}-N_{3}=m^{1}, \\
2 N_{1}+N_{2}-N_{4}=m^{1}+m^{2} .
\end{gathered}
$$

See fig. 5. Clearly we can consider more blow ups. In this way we can obtain arbitrary convex subset of integral points of $\mathbf{C}^{2}$ kept.

$\mathbf{C}^{3}$ : For our next example consider $\mathbf{C}^{3}$ blown up at a point. This corresponds to removing a point in $\mathbf{C}^{3}$ and replacing it with a $\mathbf{P}^{2}$. The embedding of $\mathbf{P}^{2}$ in the space gives $\mathcal{O}(-1) \rightarrow \mathbf{P}^{2}$. Note that again this is not a Calabi-Yau geometry (as the normal bundle is not $\mathcal{O}(-3))$. This can be realized by considering 4 variables with one charge

$$
Q=(1,1,1,-1)
$$

leading to

$$
p_{1}+p_{2}+p_{3}-p_{4}=t
$$

which can be identified with the positive octant $\left(p_{1}, p_{2}, p_{3}\right)$ with the corner removed:

$$
p_{4}=p_{1}+p_{2}+p_{3}-t \geq 0 .
$$




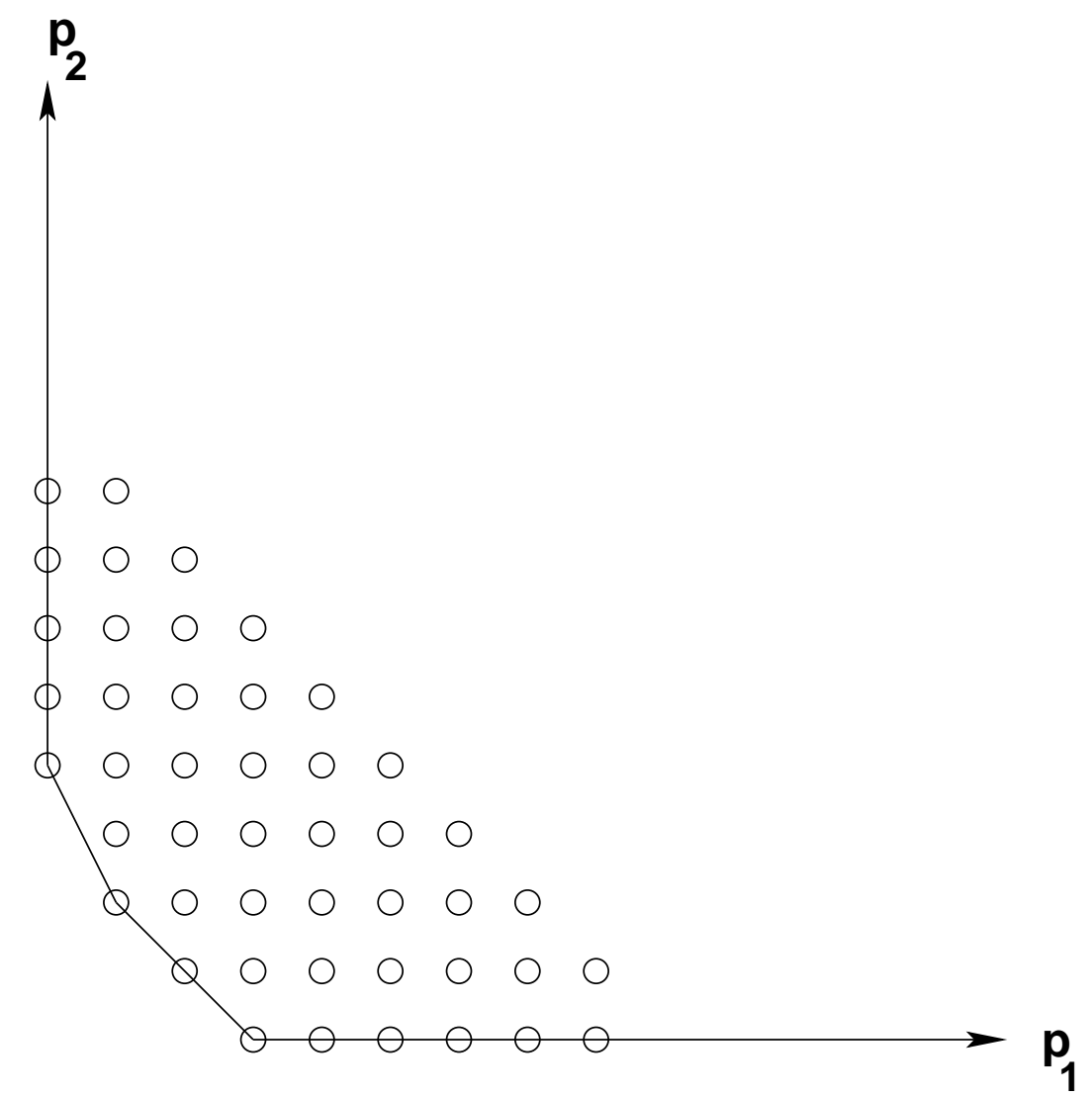

Fig. 5: The diagram shows two blow ups of $\mathbf{C}^{2}$. The diagram shown corresponds to $t^{1}=3 g_{s}$ and $t^{2}=g_{s}$.
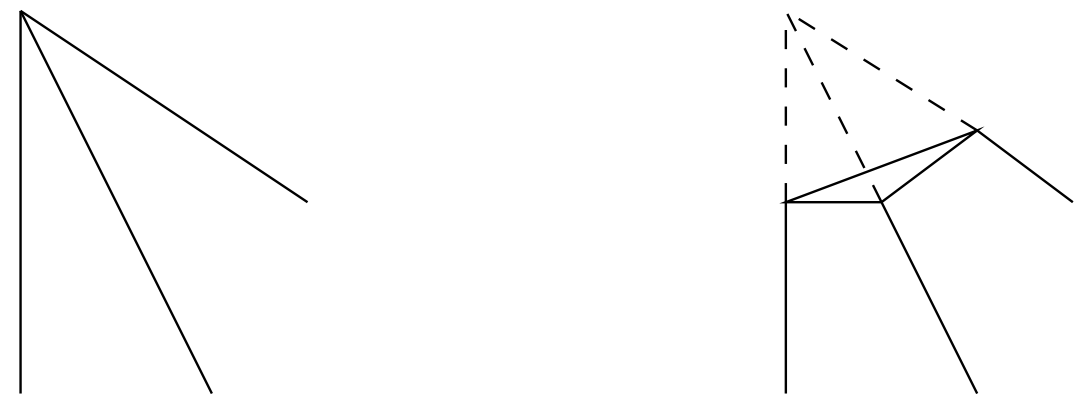

Fig. 6: Blowing up the origin of $\mathbf{C}^{3}$ corresponds to chopping off the corner of the base of $\mathbf{C}^{3}$ and replacing it with a triangle.

The corner is replaced by the triangle

$$
p_{1}+p_{2}+p_{3}=t
$$

which corresponds to $\mathbf{P}^{2}$ of size $t$ as shown in fig. 6 . 


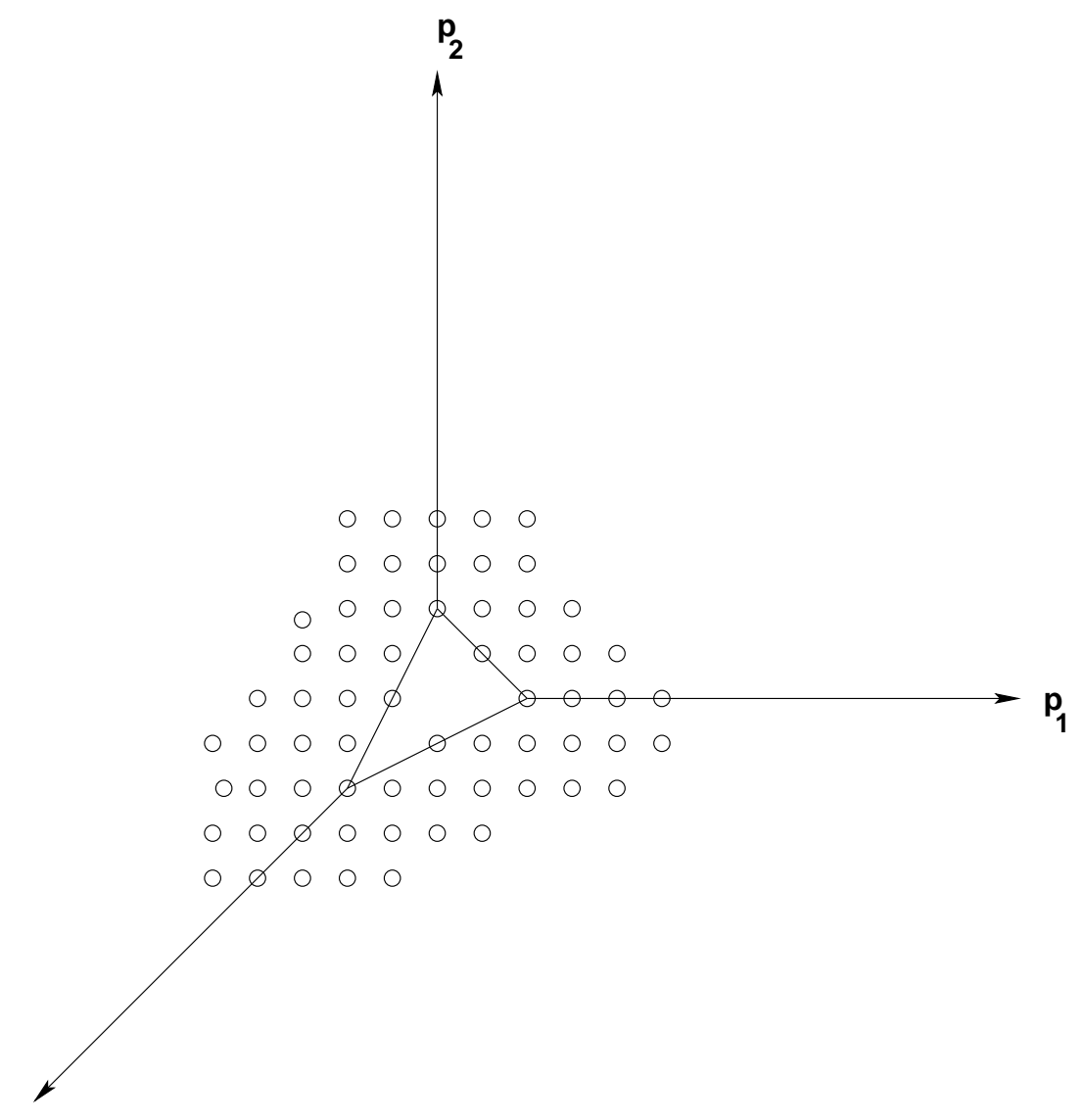

Fig. 7: The diagram shows the blowing up of a point in $\mathbf{C}^{3}$ and its replacement with $\mathbf{P}^{2}$. The case depicted corresponds to the Kähler class $t=2 g_{s}$.

Again if we consider $t$ to be quantized we have for the allowed quantum states with $N_{i} \geq 0$ :

$$
N_{1}+N_{2}+N_{3}-N_{4}=m
$$

Compared to $\mathbf{C}^{3}$ we have deleted $m(m+1)(m+2) / 6$ points (states) from the Hilbert space (see fig. 7). We shall meet this example below, in the context of noncommutative instantons.

For our next example consider two blow ups of $\mathbf{C}^{\mathbf{3}}$ : We first blow a point up to $\mathbf{P}^{2}$ and then blow up a point on the blown up $\mathbf{P}^{2}$ to a $\mathbf{P}^{1}$. This can be described by 5 variables with two set of charges

$$
\begin{aligned}
& Q^{1}=(1,1,1,-1,0), \\
& Q^{2}=(1,0,0,1,-1),
\end{aligned}
$$




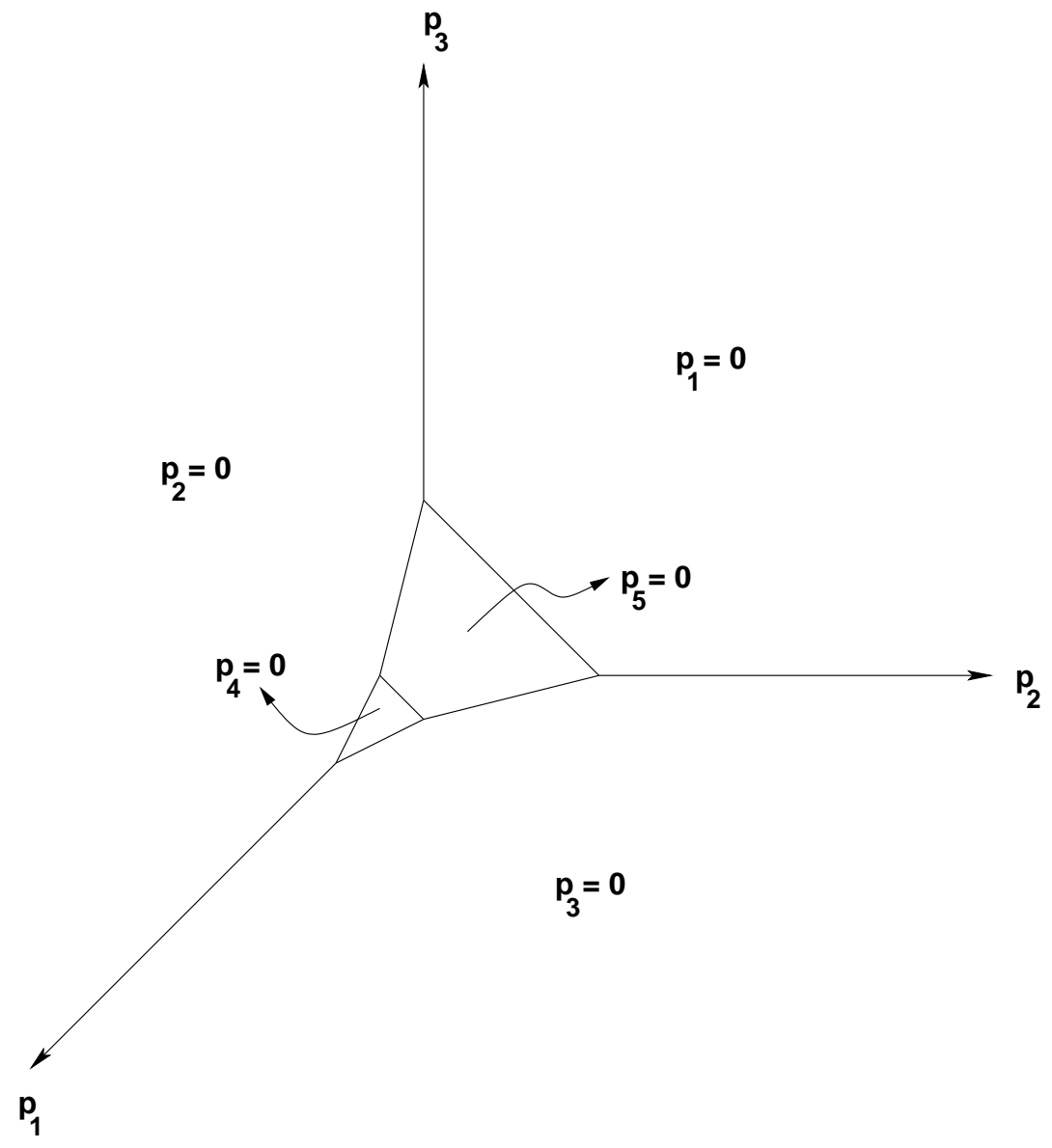

Fig. 8: The diagram shows two blow ups of $\mathbf{C}^{3}$ leading to a $\mathbf{P}^{2}$ and Hirzebruch surface $\mathbf{F}_{1}$.

which leads to

$$
\begin{gathered}
p_{1}+p_{2}+p_{3}-p_{4}=t^{1} \\
p_{1}+p_{4}-p_{5}=t^{2}
\end{gathered}
$$

This geometry is depicted in fig. 8. If $t_{1}>t_{2}$ this corresponds to the geometry of a $\mathbf{P}^{\mathbf{2}}$ connected to a Hirzebruch surface $\mathbf{F}_{\mathbf{1}}$, inside the blown up $\mathbf{C}^{3}$. Again if we consider the integral subspace the states we keep can be easily deduced.

Clearly one can continue this to more complicated blow ups. One such example is shown in fig. 9.

$\mathcal{O}(-1) \oplus \mathcal{O}(-1) \rightarrow \mathbf{P}^{1}$ : We can also consider 3d toric geometries which are not obtained by blowing up $\mathbf{C}^{3}$. For example we can consider four variables with one charge

$$
Q=(-1,1,-1,1),
$$




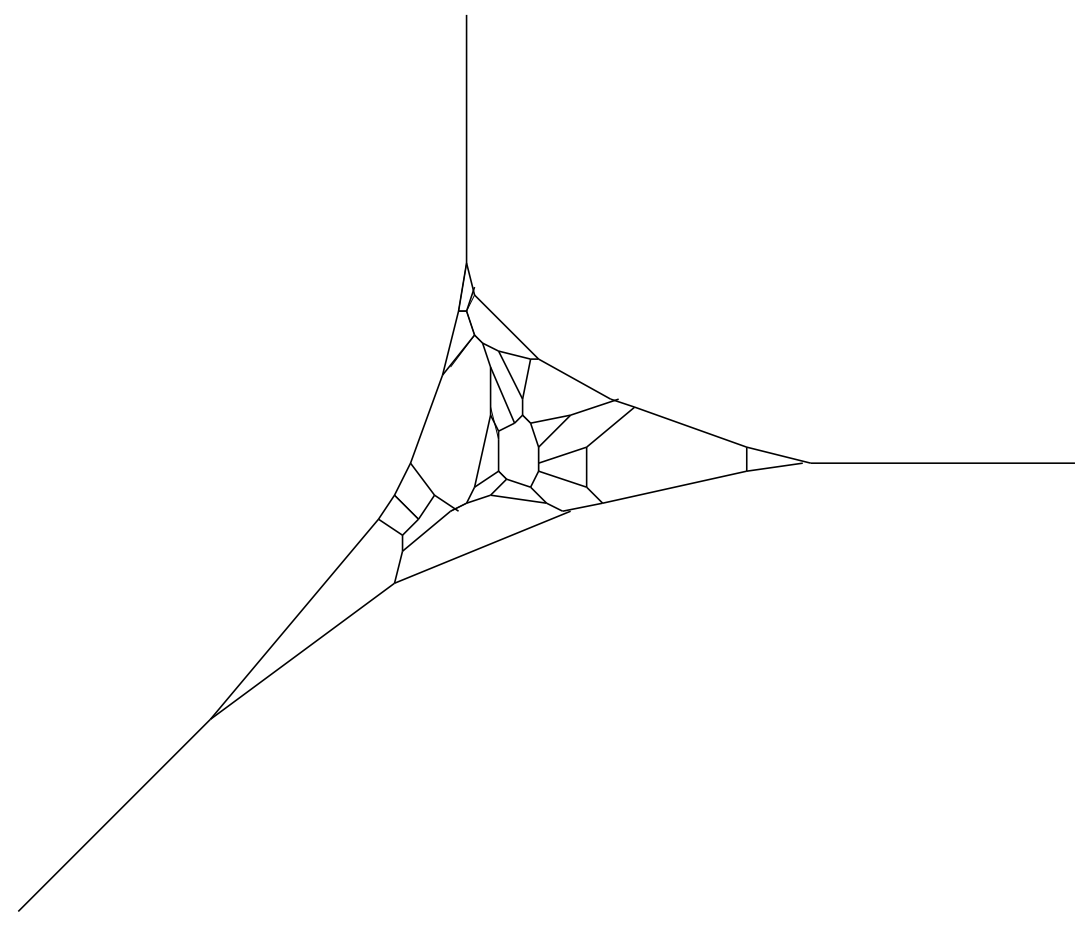

Fig. 9: We can also consider more general toric blowups of $\mathbf{C}^{3}$.

leading to

$$
-p_{1}+p_{2}-p_{3}+p_{4}=t
$$

This can be viewed as the subspace of the octant $\left(p_{1}, p_{2}, p_{3}\right)$ with the further constraint

$$
p_{4}=p_{1}-p_{2}+p_{3}+t \geq 0 .
$$

This geometry is depicted in fig. 10. Note that $p_{1}=p_{3}=0$ in this space leads to the projection of $\mathbf{P}^{1}$ of size $t$, parameterized by $\left(p_{2}, p_{4}\right)$ in the momentum space. This in fact corresponds to $O(-1) \oplus O(-1) \rightarrow \mathbf{P}^{1}$ geometry, which is a Calabi-Yau space. We can take $t=m g_{s}$ and obtain points of the Hilbert space with $N_{i} \geq 0$ satisfying

$$
N_{1}+N_{2}-N_{3}-N_{4}=m .
$$

We can also blow up points in this space. To do so we introduce one more variable (neutral under the previous charge) and consider the additional charge

$$
(1,0,1,1,-1)
$$

which blows up the point $p_{1}=p_{3}=p_{4}=0$. Or we could also blow up along $\mathbf{P}^{1}$. For example we consider instead the additional charge

$$
(0,0,1,1,-1)
$$




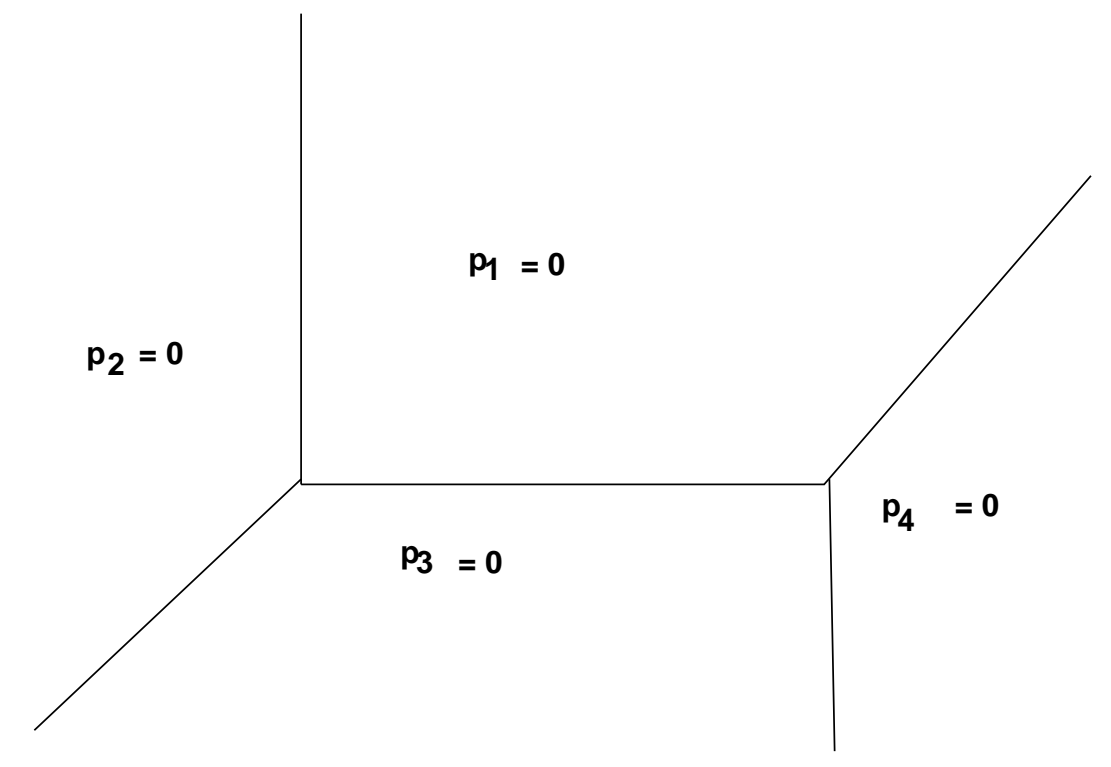

Fig. 10: The diagram shows the toric geometry of $\mathcal{O}(-1) \oplus \mathcal{O}(-1) \rightarrow \mathbf{P}^{1}$.

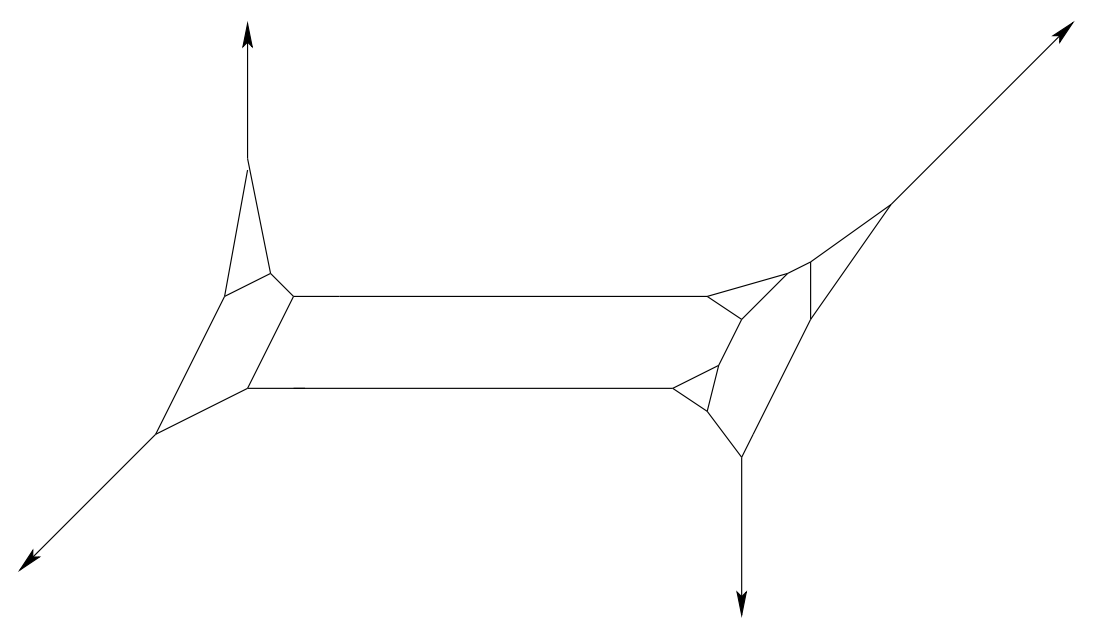

Fig. 11: The diagram shows blow ups of $O(-1) \oplus O(-1) \rightarrow \mathbf{P}^{1}$ along points at the vertices and $\mathbf{P}^{1}$ (along the edge).

We can also consider both 2 dimensional blow ups, i.e., blow ups along $\mathbf{P}^{1}$ and 3 dimensional blow up of a point. We can also consider various multi-blow ups. See as an example fig. 11.

\subsection{Toric Branes}

We will also be interested in Lagrangian A-branes which can be described by toric 


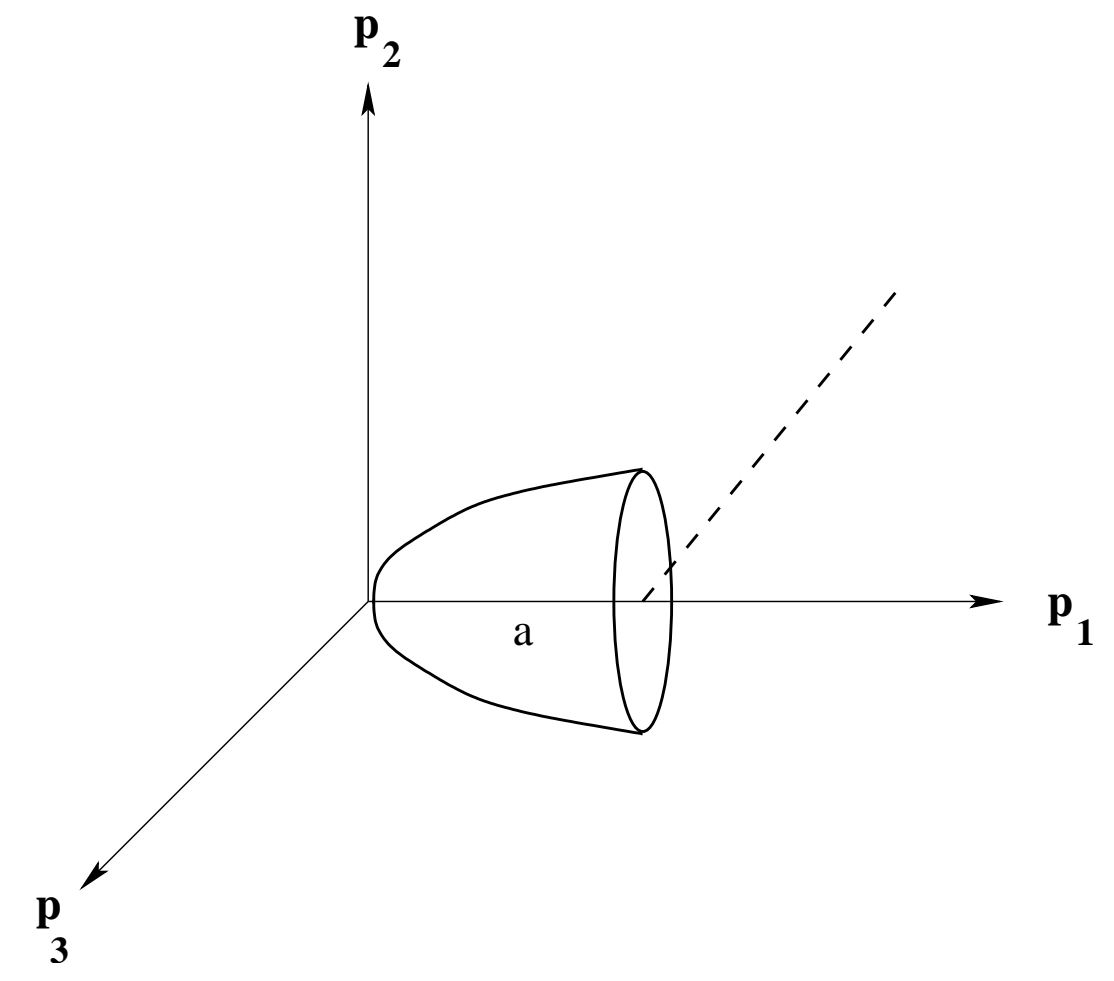

Fig. 12: The diagram shows A-brane (represented by dashed line) which projects to a half-line in the toric base. Its topology is $\mathbf{S}^{1} \times \mathbf{R}^{2}$ and there is a minimal disc with area $a$ ending on the brane (the circle comes from the fiber).

geometry. These were first studied in this context in [19] : For simplicity we only discuss the case of $\mathbf{C}^{3}$. Though this can be extended to other toric geometries, as discussed in [19]: Defining the image of a Lagrangian A-brane in the base of the toric fibration, i.e., $\left(p_{1}, p_{2}, p_{3}\right)$ space, uniquely fixes its dependence on the angular part. An interesting class of A-branes was studied in [19] which has the geometry given by

$$
p_{1}=p_{2}+a=p_{3}+a, \quad \theta_{1}+\theta_{2}+\theta_{3}=0
$$

where $a>0$ (note that $a$ has dimensions of area, as $p_{i}=\left|z_{i}\right|^{2}$ ). These branes have the topology of $\mathbf{S}^{1} \times \mathbf{R}^{2}$ and end on the $p_{1}$ axis. The moduli of these branes is fixed by the parameter $a$ which combines with the expectation value of the Wilson loop on $\mathbf{S}^{1}$ to become a complexified parameter. See fig. 12. There is a holomorphic disc in the geometry which ends on the Lagrangian submanifold. This disc projects onto the interval $0 \leq p_{1} \leq a, p_{2}=p_{3}=0$. The area of this disc is $a$. We can view this brane as the probe of $\mathbf{C}^{3}$ geometry. 


\subsection{Twisted masses}

In the linear sigma model construction of the toric Calabi-Yau $X$ one can introduce the so-called twisted masses [20,21], which deform the sigma model by some potential terms related to holomorphic isometries. In principle, one can turn on the mass for each chiral field $z^{i}, i=1, \ldots, n+r$. We shall denote them by $\epsilon_{i}$. In what follows we shall use the following vector field on $\mathbf{C}^{n+r}$ which descends to $X$ :

$$
\begin{gathered}
\Omega=\sum_{i=1}^{n+r} \epsilon_{i} \frac{\partial}{\partial \theta_{i}} \\
=\frac{i}{2} \sum_{i=1}^{n+r} \epsilon_{i}\left(z^{i} \partial_{i}-\bar{z}^{i} \bar{\partial}_{i}\right)
\end{gathered}
$$

Where $\theta_{i}$ is the phase of $z_{i}$. The vector $\frac{\partial}{\partial \theta_{i}}$ rotates the phase of $z_{i}$. Hence the fixed locus of this vector is where $z_{i}=0$. For generic masses the generic orbits of this vector field densely fill Lagrangian three-tori, which are the fibers of the projection:

$$
\vec{p}: X \rightarrow \Delta(X)
$$

The fixed points $f$ of this vector field are the vertices of $\Delta(X)$. Near each such vertex $X$ looks like a copy of $\mathbf{C}^{n}$, and $\Omega$ looks like:

$$
\begin{gathered}
\Omega \rightarrow \Omega_{f}=\sum_{\alpha=1}^{n} \epsilon_{\alpha f} \frac{\partial}{\partial \theta_{\alpha}} \\
=\frac{i}{2} \sum_{\alpha=1}^{n} \epsilon_{\alpha f}\left(z^{\alpha} \partial_{\alpha}-\bar{z}^{\alpha} \bar{\partial}_{\alpha}\right)
\end{gathered}
$$

where $\epsilon_{\alpha f}$ - the local weights - are some linear functions of $\epsilon_{i}$ and $z_{\alpha}$ are the local coordinates in terms of which the vertex is given by $z_{\alpha}=0 . \theta_{\alpha}$ are the phases of $z_{\alpha}$. For future use let us recall that the vector field $\Omega$ on the Kähler manifold $X$, with Kähler form $k_{0}$, is symplectic and is generated by some Hamiltonian $H$ :

$$
\iota_{\Omega} k_{0}=d H, \quad H=\sum_{i} \epsilon_{i} p_{i}
$$

Two fixed points $f_{1}$ and $f_{2}$ may be connected by an $\Omega$-invariant two-sphere $\mathbf{P}^{1}$, which we sometimes denote by $\mathbf{P}_{e}^{1}$, where $e=\left(f_{1}, f_{2}\right)$. Changing the order $f_{1} \leftrightarrow f_{2}$ does not change the sphere geometrically, but reverses the orientation of the rotation the vector field 


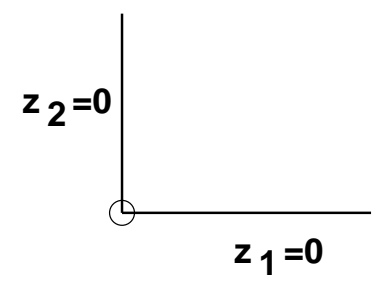

Fig. 13: The fixed point locus of $\mathbf{C}^{2}$. The small circle shows the fixed point in the geometry.

$\Omega$ induces on it. Thus one ends up with the graph $\Gamma$ with vertices $f$ and edges $e$, each edge being endowed with the pair of integers $m_{1}, m_{2}$, describing the topology of the normal bundle to the sphere. Of course, the graph $\Gamma$ is formed by the edges and the vertices of the (noncompact) polytope $\Delta(X)$ which is the image of $X$ under the $\mathbf{T}^{n}$-moment map.

Here are a few examples:

$\mathbf{C}^{2}$ : Let us denote by $\left(z_{1}, z_{2}\right)=\left(\left|z_{1}\right| e^{i \theta_{1}},\left|z_{2}\right| e^{i \theta_{2}}\right)$ the coordinates of $\mathbf{C}^{2}$. In this case the vector field is given by

$$
\Omega=\epsilon_{1} \frac{\partial}{\partial \theta_{1}}+\epsilon_{2} \frac{\partial}{\partial \theta_{2}}
$$

Its action at a point $\left(z_{1}, z_{2}\right)$ is given by

$$
\left(z_{1}, z_{2}\right) \rightarrow\left(z_{1} e^{i \epsilon_{1}}, z_{2} e^{i \epsilon_{2}}\right)
$$

Thus $\mathbf{C}^{2}$ has only one fixed point given by $\left(z_{1}, z_{2}\right)=(0,0)$. There are also two fixed planes under $\Omega$ given by $\left(z_{1}, z_{2}\right)=\left(0, z_{2}\right)$ and $\left(z_{1}, z_{2}\right)=\left(z_{1}, 0\right)$.

Thus on the $\left(\left|z_{1}\right|^{2},\left|z_{2}\right|^{2}\right)$ plane we can represent $\mathbf{C}^{2}$ as shown in fig. 13 .

$\mathbf{P}^{1}$. The linear sigma model charges for this geometry are $(1,1)$. This means that we can represent $\mathbf{P}^{1}$ as the quotient of $\mathbf{C}^{2}$ by the following action:

$$
\left(z_{1}, z_{2}\right) \rightarrow\left(s t_{1} z_{1}, s t_{2} z_{2}\right), \quad s \in \mathbf{C}^{*}
$$

The vector field $\Omega=\epsilon_{1} \frac{\partial}{\partial \theta_{1}}+\epsilon_{2} \frac{\partial}{\partial \theta_{2}}$ descends to a vector field $\widehat{\Omega}$ on $\mathbf{P}^{1}$. In the coordinate patch $z_{1} \neq 0\left(z_{2} \neq 0\right)$ the local coordinate on $\mathbf{P}^{1}$ is $\frac{z_{2}}{z_{1}}=z=|z| e^{i \theta}\left(z_{1} / z_{2}=w=|w| e^{i \phi}\right)$ and the vector field $\widehat{\Omega}$ is given by

$$
\begin{array}{ll}
\widehat{\Omega}=\frac{\partial}{\partial \theta}, & z_{1} \neq 0, \\
\widehat{\Omega}=\frac{\partial}{\partial \phi}, & z_{2} \neq 0 .
\end{array}
$$




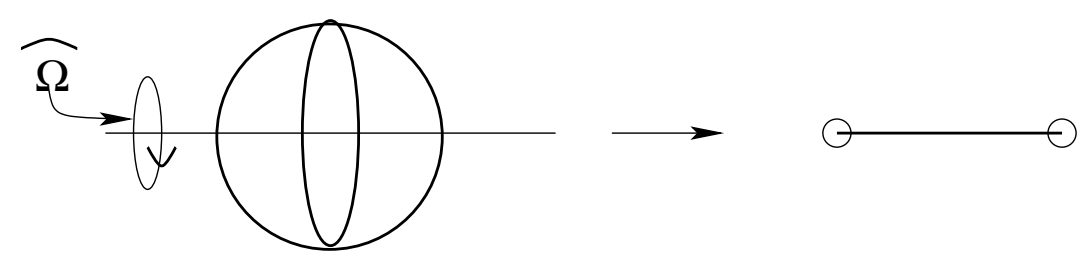

Fig. 14: The vector field $\widehat{\Omega}$ rotates the $\mathbf{P}^{1}$ around the axis passing through $z_{1}=0$ and $z_{2}=0$.

It is clear that the two fixed points of the vector field $\widehat{\Omega}$ are given by $z_{1}=0$ and $z_{2}=0$, fig. 14. The "weights" of the toric action are given by the action of $\widehat{\Omega}$ on the tangent space to the fixed points. In this case the weights are given by $\left(\epsilon_{1}-\epsilon_{2}\right)$ and $\left(\epsilon_{2}-\epsilon_{1}\right)$ corresponding to the fixed points $z_{2}=0$ and $z_{1}=0$ respectively. This follows from the fact that at the fixed point given by $z_{1}=0$ the vectors in the tangent space under the toric action given by

$$
z_{2} \rightarrow s t_{2} z_{2}
$$

transform as $v \rightarrow v e^{i\left(\epsilon_{2}-\epsilon_{1}\right)}$.

Local $\mathbf{P}^{1}$ in Calabi-Yau 3-fold: In this case, there are two fixed points, $f=N, S$ the North and the South poles of the sphere, the $\Omega$ field descends from the generic toric rotation of $\mathbf{C}^{4}$ of which the local $\mathbf{P}^{1}$ is the quotient:

$$
\left(z_{0}, z_{1}, z_{2}, z_{3}\right) \mapsto\left(\lambda t_{0} z_{0}, \lambda t_{1} z_{1}, \lambda^{-1} t_{2} z_{2}, \lambda^{-1} t_{3} z_{3}\right), \quad \lambda, t_{i} \in \mathbf{C}^{*}
$$

The action of $s \in \mathbf{C}^{*}$ on $z_{0,1,2,3}$ follows from the linear sigma model charges for this geometry given by $(1,1,-1,-1)$. The toric diagram of this geometry is shown in fig. 10 . The North pole is the point $(1: 0: 0: 0)$ and the South pole is $(0: 1: 0: 0)$. The tangent space at the North pole is $\mathbf{C}^{3}$ parameterized by $\left(z_{1}, z_{2}, z_{3}\right)$ and the tangent space at the South pole is also $\mathbf{C}^{3}$ but parameterized by $\left(z_{0}, z_{2}, z_{3}\right)$. The local weights given by the eigenvalues of the toric action on the tangent space are: at $N:\left(\epsilon_{1}-\epsilon_{0}, \epsilon_{2}+\epsilon_{0}, \epsilon_{3}+\epsilon_{0}\right)$, at $S:\left(\epsilon_{0}-\epsilon_{1}, \epsilon_{2}+\epsilon_{1}, \epsilon_{3}+\epsilon_{1}\right)$.

Non-generic $\mathbf{P}^{1}$ in Calabi-Yau 3-fold: This is the total space of the bundle $\mathcal{O}\left(-m_{1}\right) \oplus$ $\mathcal{O}\left(-m_{2}\right)$ over $\mathbf{P}^{1}$, with $m_{1}+m_{2}=2$. The condition $m_{1}+m_{2}=2$ follows from the total space being Calabi-Yau. The linear sigma model construction of this space has a single $U(1)$ gauge group with the charge vector: $\left(1,1,-m_{1},-m_{2}\right)$. This geometry can be described as a quotient of $\mathbf{C}^{4}$ by the following action:

$$
\left(z_{0}, z_{1}, z_{2}, z_{3}\right) \rightarrow\left(s z_{0}, s z_{1}, s^{-m_{1}} z_{2}, s^{-m_{2}} z_{3}\right), \quad s \in \mathbf{C}^{*} .
$$




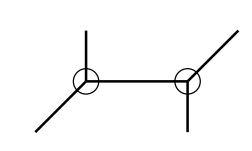

$O(-1)+O(-1)$

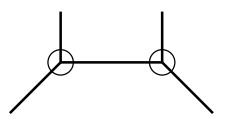

$\mathrm{O}(-2)+\mathrm{O}(0)$

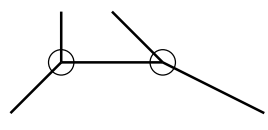

$\mathrm{O}(-3)+\mathrm{O}(1)$

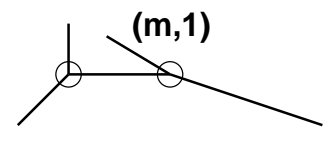

$O(-2+m)+O(-m)$

Fig. 15: The toric diagram of $\mathcal{O}(-2-m) \oplus \mathcal{O}(m)$ over $\mathbf{P}^{1}$. The two small circles show the fixed points of the geometry.

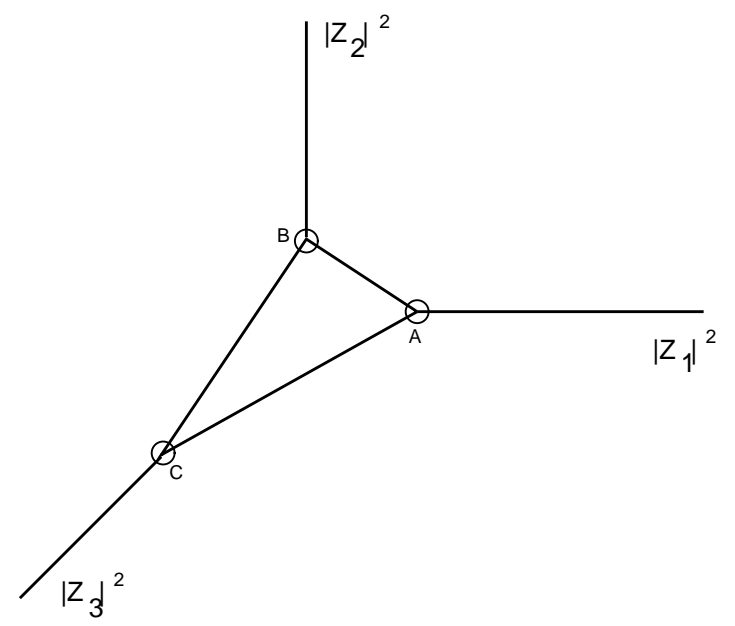

Fig. 16: Toric diagram of $\mathcal{O}\left(m_{0}\right)$ bundle over the weighted projective space $\mathbf{P}_{m_{1}, m_{2}, m_{3}}^{2}$. The fixed points of the torus action are shown by small circles.

The toric diagram is shown in fig. 15.

There are again two fixed points $f=N$ and $f=S$, with the local weights:

$$
\begin{aligned}
& \left(\epsilon_{1 N}, \epsilon_{2 N}, \epsilon_{3 N}\right)=\left(\epsilon_{1}-\epsilon_{0}, \epsilon_{2}+m_{1} \epsilon_{0}, \epsilon_{3}+m_{2} \epsilon_{0}\right) \\
& \left(\epsilon_{1 S}, \epsilon_{2 S}, \epsilon_{3 S}\right)=\left(\epsilon_{0}-\epsilon_{1}, \epsilon_{2}+m_{1} \epsilon_{1}, \epsilon_{3}+m_{2} \epsilon_{1}\right)
\end{aligned}
$$

respectively. Note that in both examples, if $\epsilon_{0}+\epsilon_{1}+\epsilon_{2}+\epsilon_{3}=0$ then $\epsilon_{1 f}+\epsilon_{2 f}+\epsilon_{3 f}=0$ for all fixed points. This is of course true for any Calabi-Yau $X$. Also, note that

$$
\left(\epsilon_{1 S}, \epsilon_{2 S}, \epsilon_{3 S}\right)=\left(-\epsilon_{1 N}, \epsilon_{2 N}+m_{1} \epsilon_{1 N}, \epsilon_{3 N}+m_{2} \epsilon_{1 N}\right)
$$

Weighted $\mathbf{P}^{2}$ in Calabi-Yau 3-fold: Let us consider the case of $\mathcal{O}\left(m_{0}\right)$ bundle over the weighted projective space $\mathbf{P}_{\left[m_{1}, m_{2}, m_{3}\right]}^{2}$ with weights $\left(m_{1}, m_{2}, m_{3}\right)$.

The toric diagram is shown in fig. 16. The linear sigma model charges for this case are given by $\left(m_{0}, m_{1}, m_{2}, m_{3}\right)$,

$$
\left(z_{0}, z_{1}, z_{2}, z_{4}\right) \mapsto\left(s^{m_{0}} t_{0} z_{0}, s^{m_{1}} t_{1} z_{1}, s^{m_{2}} t_{2} z_{2}, s^{m_{3}} t_{3} z_{3}\right) .
$$


This geometry has three fixed points $\{A, B, C\}$ are given by $\left(z_{0}, z_{1}, z_{2}, z_{3}\right)=$ $(0,1,0,0),(0,0,1,0),(0,0,0,1)$ respectively. These three points are not smooth but rather have orbifold singularities of order $m_{1}, m_{2}, m_{3}$. The corresponding weights $\epsilon_{\alpha f}$ can be calculated using (3.11),

$$
\begin{aligned}
& \left(\epsilon_{1 A}, \epsilon_{2 A}, \epsilon_{3 A}\right)=\left(\epsilon_{0}-\frac{m_{0}}{m_{1}} \epsilon_{1}, \epsilon_{2}-\frac{m_{2}}{m_{1}} \epsilon_{1}, \epsilon_{3}-\frac{m_{3}}{m_{1}} \epsilon_{1}\right) \\
& \left(\epsilon_{1 B}, \epsilon_{2 B}, \epsilon_{3 B}\right)=\left(\epsilon_{0}-\frac{m_{0}}{m_{2}} \epsilon_{2}, \epsilon_{1}-\frac{m_{1}}{m_{2}} \epsilon_{2}, \epsilon_{3}-\frac{m_{3}}{m_{2}} \epsilon_{2}\right) \\
& \left(\epsilon_{1 C}, \epsilon_{2 C}, \epsilon_{3 C}\right)=\left(\epsilon_{0}-\frac{m_{0}}{m_{3}} \epsilon_{3}, \epsilon_{1}-\frac{m_{1}}{m_{3}} \epsilon_{3}, \epsilon_{2}-\frac{m_{2}}{m_{3}} \epsilon_{3}\right)
\end{aligned}
$$

Note that the sum of weights at each fixed point are such that $\sum_{\alpha=1}^{3} \epsilon_{\alpha f}=0(f=$ $A, B, C)$ if $\epsilon_{0}+\epsilon_{1}+\epsilon_{2}+\epsilon_{3}=0$ and $m_{0}+m_{1}+m_{2}+m_{3}=0$. The latter condition is required for the total space to be a Calabi-Yau threefold.

Let us now return to the linear sigma model. The Calabi-Yau condition $\sum_{i} Q_{i}^{a}=0$ entails a natural constraint on the choice of twisted masses, or weights:

$$
\sum_{i=1}^{3+r} \epsilon_{i}=0
$$

It follows that at each fixed point $f$ the local weights sum to zero: $\epsilon_{1 f}+\epsilon_{2 f}+\epsilon_{3 f}=0$.

\section{Application to $\mathbf{C}^{3}$}

In section 2 we proposed that the target space description of topological A-model can be viewed as sums over distinct Kähler manifolds, subject to quantization of Kähler moduli in units of $g_{s}$. In section 3 we gave examples of toric blow ups of $\mathbf{C}^{3}$. In this section we propose that for toric geometries, such as $\mathbf{C}^{3}$, the sum over distinct Kähler manifolds localizes on distinct toric geometries. This we conjecture to be the case at least as long as one is probing the geometry with toric branes. A natural way this can be accomplished is proposed in section 6. As far as the discussion in this section and section 5 is concerned we assume such a localization holds with a simple measure and postpone a technical justification of this assumption to section 6 . In fact we propose following (2.1)

$$
Z=\sum_{\text {quantized toric blow ups }} \exp \left(N g_{s}\right)
$$


where $N$ denotes the number of holomorphic sections of the bundle whose curvature is $k / g_{s}$.

We can also turn the question around: We ask which configuration of sections, and thus which values of $N$ are allowed? If $|\psi\rangle \in \mathcal{H}$ is a holomorphic section, then so is

$$
z_{1}^{n_{1}} z_{2}^{n_{2}} z_{3}^{n_{3}}|\psi\rangle
$$

for $n_{i} \geq 0$. This is because we are not changing the complex structure of $\mathbf{C}^{3}$ but only blowing up the geometry, which modifies its Kähler geometry. If we denote the state $|\psi\rangle=\left|m_{1}, m_{2}, m_{3}\right\rangle$, then as discussed before the above action implies that

$$
\left|m_{1}, m_{2}, m_{3}\right\rangle \in \mathcal{H} \rightarrow\left|m_{1}+n_{1}, m_{2}+n_{2}, m_{3}+n_{3}\right\rangle \in \mathcal{H}
$$

as long as $n_{i} \geq 0$.

The set of such $\mathcal{H}$ is in one to one correspondence with 3D Young diagrams. Counting the states of such $\mathcal{H}$ compared to that of $\mathbf{C}^{3}$ can be viewed as counting the negative of the number of boxes of the 3D Young diagram. Thus if we restrict to this set we precisely obtain the partition associated with crystal melting, but now viewed as summing up over various Kähler geometries, obtained by toric blowups of $\mathbf{C}^{3}$. This is in fact not exactly true, because we have not shown that all Kähler geometries of toric blow ups give rise to such $\mathcal{H}$. Indeed this is not the case: As we discussed before the points on the toric base always form a convex set. But this is not necessarily true for all $\mathcal{H}$. For example, consider a 3d Young diagram given by a cube of size $N$. The complement of the integral points on the octant is not a convex set, thus it does not correspond to a geometric blow up of $\mathbf{C}^{3}$. It should be clear from our discussion of toric geometry in section 2 that a 3D Young diagram corresponds to a blow up geometry if and only if its complement is a convex set. Given the precise match which was obtained from the 3d Young diagram partition function and topological string in [9] it is natural to postulate that we should extend the space of geometries we are summing over to include such cases of 'non-geometric excitations' of $\mathbf{C}^{3}$. These 'non-geometric' excitations turn out to correspond to excitations of the gauge theory discussed in section 6 , which define the target space topological gravity. That one may need to sum over non-geometric excitations to obtain a consistent quantum theory of gravity was already observed in three dimensional gravity in [11] where it was seen that certain configurations of $S L(2, \mathbf{C})$ Chern-Simons connection correspond to negative values of $\operatorname{det}(g)$. This is probably not unrelated to the convexity/concavity we have found here, 
which distinguishes geometric versus non-geometric excitations of $\mathbf{C}^{3}$ : In fact the A-model Kähler gravity induces on Lagrangian submanifolds the $S L(2, \mathbf{C})$ Chern-Simons gravity theory [22].

Thus we see that with these assumptions, the sum over generalized discrete toric geometries contributes

$$
Z=\sum_{\pi} q^{|\pi|}
$$

where $q=\exp \left(-g_{s}\right)$, and $\pi$ denotes a 3D young diagram (or equivalently a generalized toric geometry), and $|\pi|$ the number of boxes in the Young diagram. Thus we have mapped the sum over target Kähler geometries to the statistical mechanics of crystal melting. We now turn to the physical interpretation of this result.

\subsection{Physical Interpretation of the Result}

We have thus mapped topological A-model Kähler gravity to a 3D cubic crystal melting problem, where the crystal occupies the positive octant. Moreover the lattice spacing is $g_{s}$. As the crystal melts we remove atoms, starting from the corner. The allowed configurations are such that the remaining atoms form a crystal with arbitrary positive translations in the three directions occupied by an atom. Each such configuration receives a thermodynamic weight factor of $q^{N}$ where $N$ is the number of atoms removed from the crystal. This statistical mechanical model has been studied in [23]. The result is that for $g_{s}<<1$ (i.e. high temperature for the crystal) the corner of the crystal gets molten up to a size of order 1 (in string units).

In particular the boundary of the crystal becomes a smooth object at the string scale, known as the limit shape (fig. 17), defined by

$$
\left(p_{1}, p_{2}, p_{3}\right)=(u+R(u, v), v+R(u, v), R(u, v))
$$

where

$$
R(u, v)=\frac{1}{4 \pi^{2}} \int d \theta d \phi \log \left|e^{-u+i \theta}+e^{-v+i \phi}+1\right|
$$

Note that the transition region of smooth (flat) and curved boundary of the crystal are defined by three curves intersecting the three planes given by $\pm e^{-u} \pm e^{-v}=1$ (excluding the case where both terms have a minus sign). 


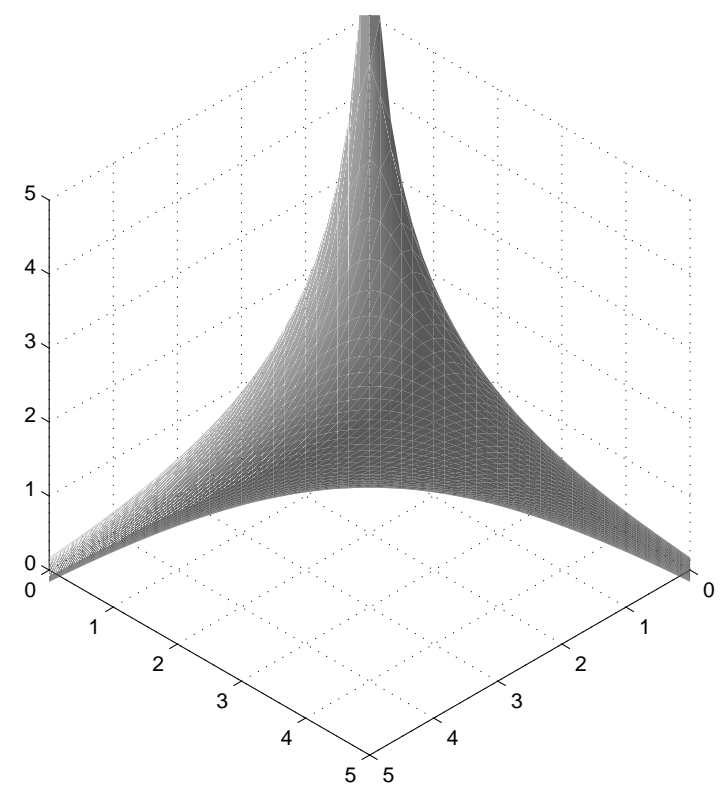

Fig. 17: The limit shape of 3d partitions.

The limit shape is the boundary of the base of $\mathbf{C}^{3}$ in the limit of very large number of blowups. It is essentially the region which replaces the origin in $\mathbf{C}^{3}$, in the limit where $g_{s} \rightarrow 0$ where the number of blowups approach infinity, connected to the rest of the flat planes of the octant. For a finite but small $g_{s}$ there are a finite but large number of faces of the blownup geometry, and the boundary is a piecewise linear version of the limit shape made up of polygons. The limit shape has the same overall size, of the order of string scale, independent of $g_{s}$. These various polygons are the compact four cycles in the blowup geometry. The convex hull of the points closest to the limit shape is the piecewise linear geometry we mentioned before. The points also define a $3 \mathrm{~d}$ partition. The fig. 18 shows the $3 \mathrm{~d}$ partition and fig. 19 shows the corresponding blownup geometry for $g_{s} \approx 0.02$.

From the viewpoint of topological string we should identify the region occupied by the lattice as the toric base of the geometry. The fact that the boundary is deformed from the naive classical picture, is consistent with the fact that worldsheet instantons modify the A-model target geometry at string scale, captured by a classical mirror geometry. In fact, as noted in [9] the boundary of the molten crystal can be viewed as a special lagrangian submanifold, which is captured by the mirror geometry [21,24] encoded by the Calabi-Yau hypersurface

$$
e^{-u}+e^{-v}+1=z w
$$




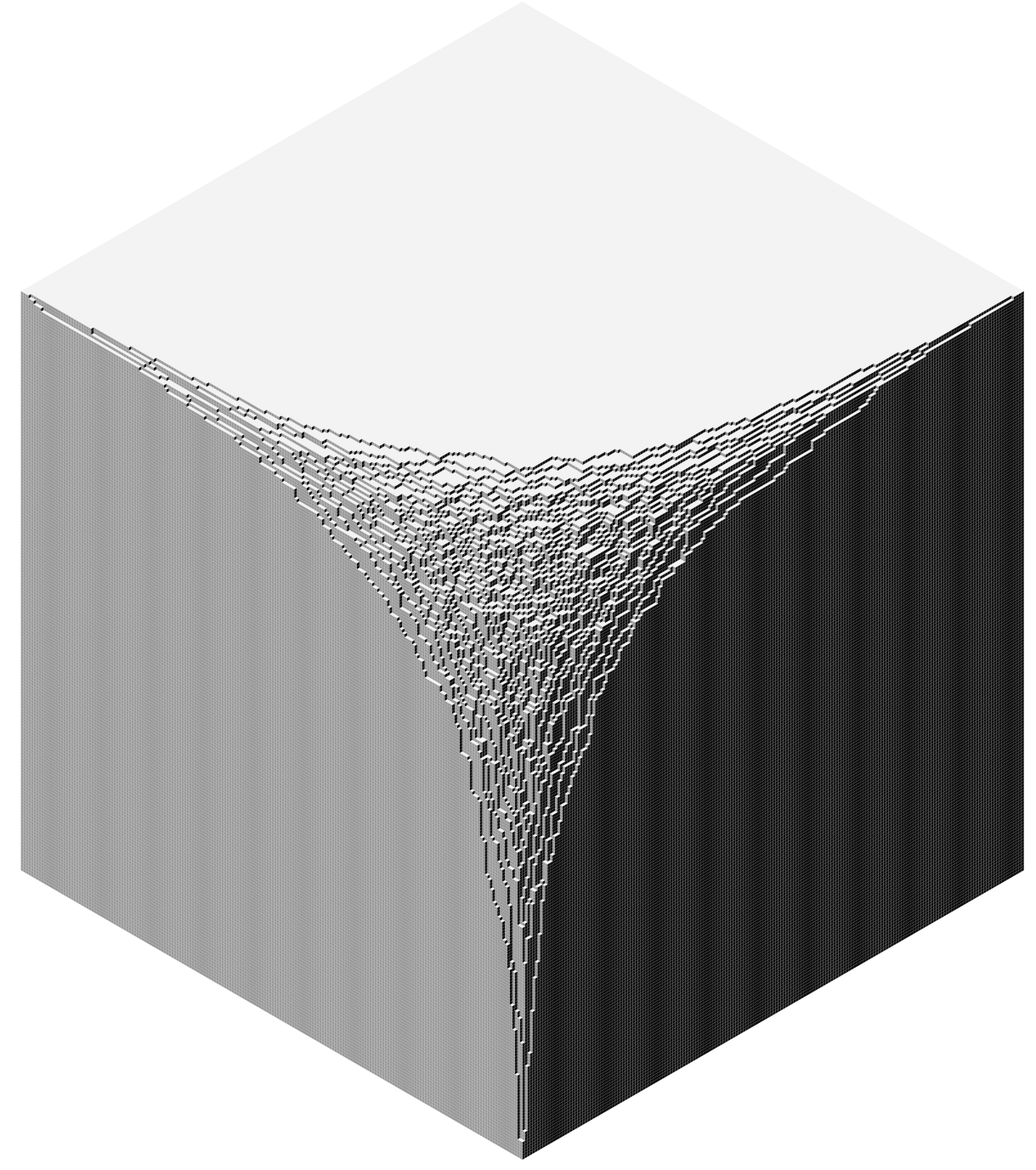

Fig. 18: A random partition with $g_{s} \approx 0.02$ inside a box of size $200 \times 200 \times 200$ lattice lengths.

The points of the blown up geometry are now deleted from space. Thus the molten piece of crystal also represents the "molten space".

Let us probe this geometry by toric Lagrangian A-branes. As discussed in section 2, we consider non-compact A-branes which project to an infinite diagonal semi-line intersecting the $p_{1}$ axis at the point $a$. Note that we are working in the string scale and $a$ has units of area. In other words in the formulae below by $a$ we mean the dimensionless quantity $a / l_{s}^{2}=a / \alpha^{\prime}$. 


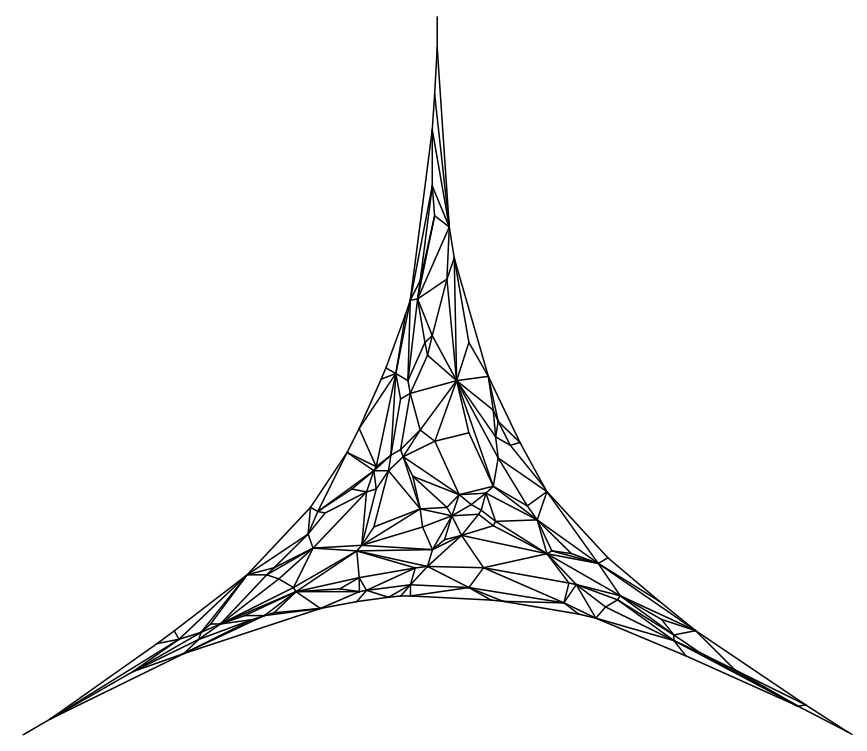

Fig. 19: The blownup geometry corresponding the 3d partition shown in fig. 18.

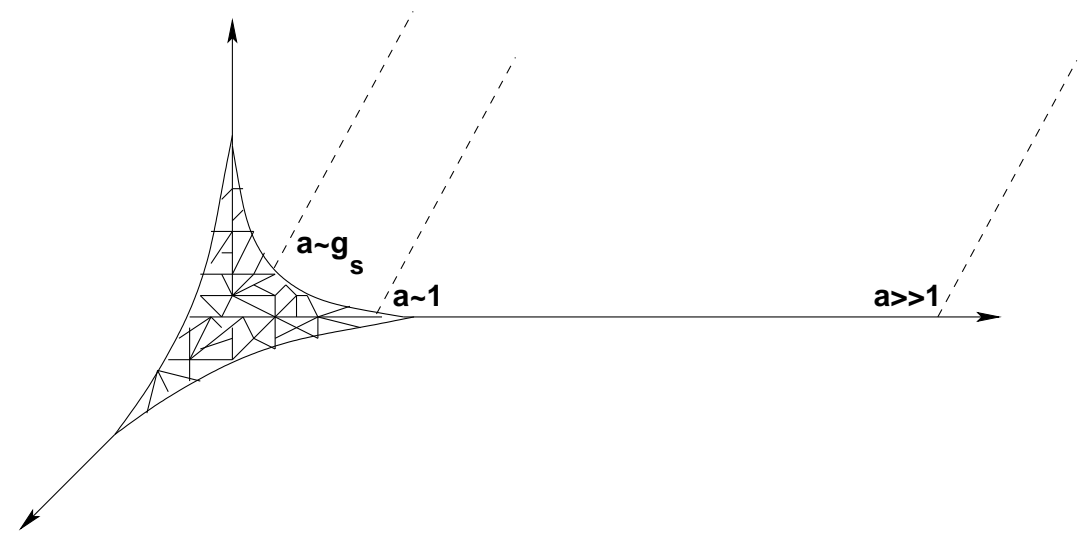

Fig. 20: As the A-brane probe moves there are three regimes of geometry. For $a>>1$ it is classical. For $a \sim 1$ it is stringy geometry and for $a \sim g_{s}$ it is a foamy geometry. The tree level worldsheet instanton corrections can be read off from the geometry of the three curves bounding the curved portion of the limit shape.

We have three distinct regions for this probe (see fig. 20):

$$
\begin{gathered}
a>>1 \quad \text { classical regime } \\
a \sim 1 \quad \text { stringy regime } \\
a \sim g_{s} \quad \text { quantum foam regime }
\end{gathered}
$$


For $a>>1$ the melting of the crystal is irrelevant and we just see the full octant. This is consistent with the fact that in this region the worldsheet instantons are highly suppressed $e^{-a} \sim 0$, and we recover the classical geometry of $\mathbf{C}^{3}$. For $a \sim 1$ we come close to the boundary of the crystal and we see the limit shape. We see this as a smooth geometry at this scale. This is the Lagrangian boundary of the space. In this regime the worldsheet instantons become relevant and give rise to the stringy geometry which is captured by the limit shape. In this regime it is important to note that the brane probe position is not strictly on the $p_{1}$ axis as that has molten away. Rather its end is replaced by the boundary regions of the limit shape, say $e^{-u}+e^{-v}=1$, where

$$
\begin{aligned}
& u=p_{1}-p_{3} \\
& v=p_{2}-p_{3}
\end{aligned}
$$

In other words in the classical regime the Lagrangian A-brane defined by $u=a, v=0$ was denoting the moduli of the brane. In the quantum theory the moduli of brane is projected to $u, v$ satisfying

$$
e^{-u}+e^{-v}=1
$$

Note that for $u=a>>0, v=0$ this is satisfied. But for $a \sim 1$ the allowed $u, v$ change. The leading string correction to the partition function (i.e. the disc amplitude) due to the presence of the brane was computed from this curve [19] and is given by

$$
F_{0}=\frac{-1}{g_{s}} \int^{a} v(u) d u=\frac{1}{g_{s}} \sum_{n>0} \frac{e^{-n a}}{n^{2}} .
$$

Note that $e^{-a}$ represents the amplitude for the disc instanton ending on the probe and $n$ labels the multicoverings of this instanton. Note that for small $a$ this diverges as $F_{0}=$ $\frac{1}{g_{s}} a \log a$. One can see that as $a \rightarrow 0$ the string perturbative expansion diverges. In fact as shown in 25] the full amplitude can be computed using the duality between Chern-Simons on $S^{3}$ and topological strings on resolved conifold [5] and is given by

$$
F=\sum_{n>0} \frac{e^{-n a}}{2 n \sinh \left(\frac{n g_{s}}{2}\right)}
$$

and expanding in powers of $g_{s}$ we find that for small $a$

$$
F_{g} \sim c_{g} g_{s}^{2 g-1} / a^{2 g-1}
$$


We thus see that as $a \sim g_{s}$ the string perturbation theory breaks down. This is the regime where we see the quantum foam and the fluctuations of the Kähler geometry. At this scale the geometry is fluctuating rather wildly and the string expansion does break down. Nevertheless the exact expression continues to hold (with a natural analytic continuation) even in this regime. This expression can also be written in the more suggestive form [26]

$$
Z=e^{F}=\prod_{n \geq 0}\left(1-e^{-a} q^{n+\frac{1}{2}}\right)
$$

In fact, as explained in [26] this satisfies a difference equation involving shifting $a \rightarrow a+g_{s}$ :

$$
Z\left(a+g_{s}, g_{s}\right)=\left(1-e^{-a} q^{\frac{1}{2}}\right) Z\left(a, g_{s}\right)
$$

which is a reflection of the integral lattice structure at the scale of $\delta a \sim g_{s}$.

Note that the foamy nature of spacetime takes place at all points, and not just the origin of $\mathbf{C}^{3}$, as it may appear from the discussion above. Toric brane probes are sensitive only to the fluctuations of geometry near the origin. Origin of $\mathbf{C}^{3}$ is the only place where the area of the disc which ends on them becomes zero and the branes become classically singular. Of course by defining the toric actions differently we get different toric branes which probe the foamy structure of $\mathbf{C}^{3}$ at other points.

So far our discussion has been in the context of $g_{s}<<1$. However we can use the lattice picture to study the regime of large $g_{s}>>1$. This is the low temperature phase for our crystal. At this point none of the atoms of the crystal have molten and so the geometry is just the classical geometry of the octant. It may appear surprising that in the extreme large string coupling constant the classical geometry dominates-even the worldsheet instantons do not survive. To make this more intuitive, note that even though the action seems to allow arbitrary fluctuations when $g_{s}>>1$ (being given by $\int k^{3} / g_{s}^{2}$ ), what suppresses fluctuations is the quantization of $k$ in units of $g_{s}: k=N g_{s}$. Effectively what this means is that if we want to blow up a point in $\mathbf{C}^{3}$ we have to replace it by a big $\mathbf{P}^{2}$ of the size $g_{s}$. This should be clearly suppressed. This is also somewhat similar to what one sees in superstring dualities where large string couplings lead to a classical regime. Note that the exact answer (4.1) is consistent with all the corrections disappearing in this limit. As $g_{s} \rightarrow \infty$, we have $q \rightarrow 0$ and $Z \rightarrow 1$. 


\section{More General Toric Geometries and the Topological Vertex}

In this section we will discuss some more general non-compact toric Calabi-Yau threefolds. For these more complicated geometries, which may have compact 2-cycles and 4-cycles, the general idea, discussed in section 2, of summing over toric blow ups with quantized Kähler classes continues to hold. The extra ingredient due to the presence of compact 2-cycles is that we can, in addition to blowing up points, also blow up along the 2-cycles. However, the restriction to toric blow ups implies that the blown up 2-cycles must be invariant under the torus action and hence must be rational curves joining the fixed points. From our discussion of toric geometries it is clear that we can view them as obtained by trivalent vertices connected by edges. Thus the choices of the blow up geometries along edges localizes to the choice of a $2 \mathrm{~d}$ Young diagram $\mu_{i}$ for each edge. If we associate to each edge a Kähler class $t_{i}=N_{i} g_{s}$ with $Q_{i}=e^{-t_{i}}$, as discussed in section 2 we will get a factor

$$
\prod_{i} Q_{i}^{\left|\mu_{i}\right|}
$$

where $\left|\mu_{i}\right|$ is the number of points associated to the Young diagram $\mu_{i}$. Note that $N_{i}\left|\mu_{i}\right|$ are the number of points being deleted from the integral points of the toric base when we blow up the $i$-th edge along the Young diagram $\mu_{i}$ and

$$
e^{-g_{s} N_{i}\left|\mu_{i}\right|}=Q_{i}^{\left|\mu_{i}\right|}
$$

represents the deficit contribution of the removal of these points to the partition function. Note that this formula can also be viewed as $Q_{i}^{c h_{2}\left(\mu_{i}\right)}$, consistent with our discussion of section 2. On top of this the toric geometry may have excitation along the vertices. These get weighted with $q^{c h_{3}}$ for each vertex. Note that for each trivalent vertex these excitations are on top of the choices of the blow ups along the three edges ending on the vertex, which are labelled by three $2 \mathrm{~d}$ Young diagrams $\mu, \nu, \lambda$. The allowed excitation of the bundle with these fixed choices gets identified with the crystal partition function with three asymptotes $\mu, \nu, \lambda$ and this leads to the topological vertex $C_{\mu, \nu, \lambda}$ [14] as shown in [9]. This is again, assuming as before, that we enlarge the space of geometries to include non-geometric excitations. The technical meaning of this assumption from the viewpoint of the $U(1)$ gauge theory instantons will be discussed in section 6 . Note that we get one factor of topological vertex for each vertex. Together with the edge contribution discussed above, this reproduces the topological vertex sum rule of [14] for computation of A-model topological amplitudes on toric CY 3-folds. 
There are two subtleties in this. One is the framing factor. This has been carefully considered in [9] and it just is there to ensure the correct number of points are counted in the partition sum, coming from the fact that different corners of the toric geometry are oriented differently. In addition there is some \pm sign factors in the gluing rule of [14]. This can be absorbed into the definition of what $Q_{i}$ are (see also our discussion in section 6 for the meaning of these sign factors).

As an example consider $\mathcal{O}(-1) \oplus \mathcal{O}(-1) \rightarrow \mathbf{P}^{1}$ : The toric diagram of this geometry is shown in fig. 10. As discussed in section 2 the partition function in this case can be written as

$$
Z=\sum e^{\mathcal{S}}=\sum_{\text {line bundles on toric blowups }} q^{c h_{3}} Q^{c h_{2}}
$$

Where $t=-\log (Q)$ is the Kähler parameter of the $\mathbf{P}^{1}$ and $c h_{2}, c h_{3}$ are Chern characters of the $U(1)$ bundle on (blown up) $X$. Note that the term involving $c h_{2}$ was absent in the $\mathbf{C}^{3}$ case; this was due to the fact that $\mathbf{C}^{3}$ had no compact 2-cycles.

To evaluate the above partition function note that in the case of $\mathbf{C}^{3}$ the contribution to $c h_{3}$ were in one to one correspondence with 3D Young diagrams. In this case also we have a correspondence between $c h_{3}$ and the $3 \mathrm{D}$ Young diagrams but in this case $c h_{3}$ is determined by a pair of 3D Young diagrams $\left(\pi_{1}, \pi_{2}\right)$ such that $c h_{3}=\left|\pi_{1}\right|+\left|\pi_{2}\right|$. This is due to the fact that there are two fixed points under the $U(1)^{3}$ action. In the melting crystal picture this corresponds to removing integral points from both corners of this crystal. If we take $Q \mapsto 0$ we essentially get two copies of $\mathbf{C}^{3}$ and the partition function is the square of the $\mathbf{C}^{3}$ partition function,

$$
Z(Q \mapsto 0)=\sum_{\pi_{1}, \pi_{2}} q^{\left|\pi_{1}\right|+\left|\pi_{2}\right|}
$$

However, for non zero $Q$ the crystal has an edge also connecting the two corners. The integral points along this edge can also be removed corresponding to blowing up the entire $\mathbf{P}^{1}$. This has the effect of replacing the neighbourhood of $\mathbf{P}^{1}$ with $\mathbf{P}^{1} \times\left(\right.$ Blow up of $\left.\mathbf{C}^{2}\right)$. 


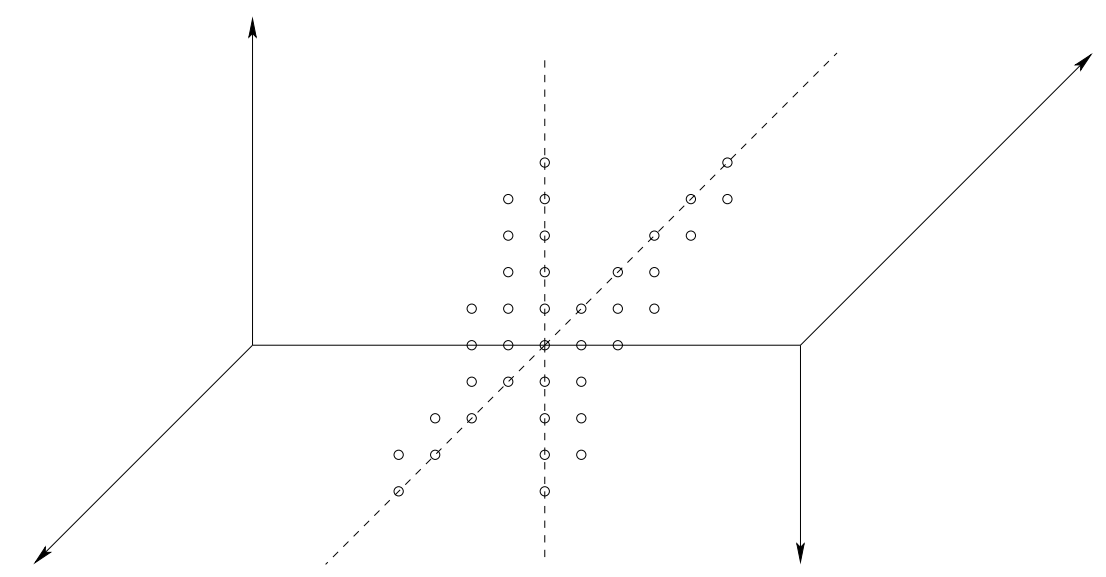

Fig. 21: More precisely the 2D partitions correspond to blowing up the entire $\mathbf{P}^{1}$.

It is easy to see that blowing up the entire $\mathbf{P}^{1}$ a number of times is in one to one correspondence with 2D Young diagrams whose complement is convex fig. 21. Thus as in the case of $\mathbf{C}^{3}$ we not only have to consider non-geometric excitation corresponding to 3D Young diagrams whose complement is not convex, but also have to consider the non-geometric excitations corresponding to 2D Young diagrams whose complement is concave. Thus the contribution to $c h_{2}$ is in one to one correspondence with 2D Young diagrams essentially because the $\mathbf{P}^{1}$ is the fixed point locus of the $U(1)^{2}$ action. Since the vertices are also invariant under this $U(1)^{2}$ action the 2D Young diagram must be compatible with the 3D Young diagrams coming from the vertices. This is also reflected by the fact that it is not possible to blow up the entire $\mathbf{P}^{1}$ without blowing up the corners as well. This implies that the 2D Young diagram, $\lambda$, must be one of the asymptotes of the 3D Young diagrams in the direction given by the $\mathbf{P}^{1}$. Thus the partition function becomes

$$
\begin{aligned}
& Z_{X}=\sum_{\lambda, \pi_{1}, \pi_{2}} q^{\left|\pi_{1}\right|+\left|\pi_{2}\right|+\left(|| \lambda||^{2}+|| \lambda^{t}||^{2}\right) / 2} Q^{|\lambda|} \\
& =\sum_{\lambda} Q^{|\lambda|} \sum_{\pi_{1}, \pi_{2}} q^{\left|\pi_{1}\right|+\left|\pi_{2}\right|+\left(|| \lambda||^{2}+|| \lambda^{t}||^{2}\right) / 2}
\end{aligned}
$$

Where the factor involving $\|\lambda\|^{2}\left(=\sum_{i} \lambda_{i}^{2}\right)$ in the above equation is due to counting of extra points arising due to perpendicular slicing (see [9]) of the 3D Young diagrams associated with the two vertices, fig. 21. As shown in fig. 21 the slicings can be rotated into each other by first rotating one of them along the first column of the $2 \mathrm{~d}$ partition $\lambda$ by $\pi / 4$ and then rotating it along the first row of $\lambda$ by $\pi / 4$. Rotation around the column 
a)

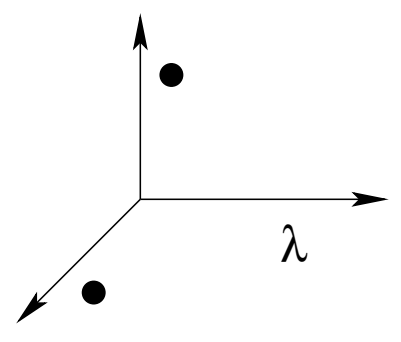

b)

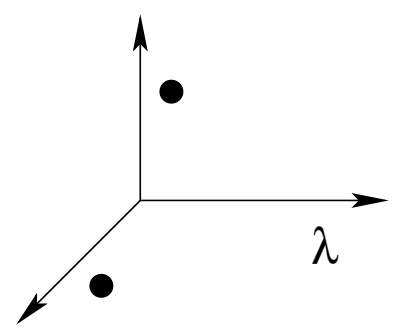

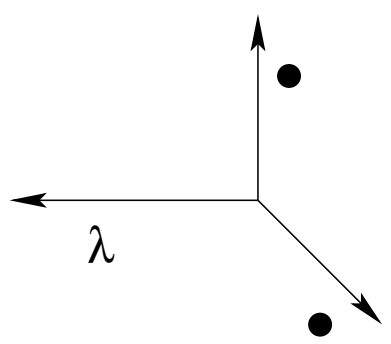

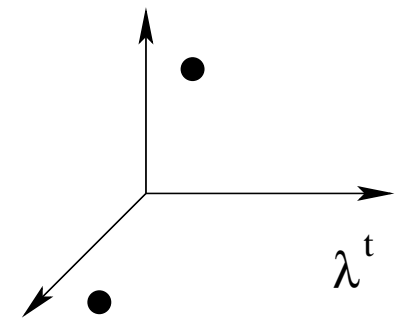

Fig. 22: a) The $3 D$ Young diagrams associated with the vertices have asymptote $\lambda$ along the $\left.\mathbf{P}^{1}, b\right)$ in terms of the topological vertex this implies a gluing rule which involves taking the transpose.

gives an extra volume $\frac{\|\lambda\|^{2}}{2}$ and rotation around the row gives an extra volume $\frac{\left\|\lambda^{t}\right\|^{2}}{2}$. The sum over 3D Young diagrams $\left(\pi_{1}, \pi_{2}\right)$ in the above equation is restricted by the condition that their asymptotics in the direction of $\mathbf{P}^{1}$ is $\lambda$.

This sum can be evaluated in terms of the topological vertex, $C_{\mu, \nu, \lambda}$, using the results of [9] (see fig. 22),

$$
\sum_{\pi_{1}, \pi_{2}} q^{\left|\pi_{1}\right|+\left|\pi_{2}\right|+\left(\| \lambda||^{2}+\left.|| \lambda^{t}\right|^{2}\right) / 2}=M(q)^{2} C \cdot \bullet \lambda\left(q^{-1}\right) C \bullet \bullet \lambda^{t}\left(q^{-1}\right) .
$$

Where $M(q)=\prod_{n>0}\left(1-q^{n}\right)^{-n}$ and $\bullet$ is the empty partition. Thus the partition function is given by

$$
\begin{gathered}
Z=M(q)^{2} \sum_{\lambda} Q^{|\lambda|}(-1)^{|\lambda|} C \cdot \bullet \lambda\left(q^{-1}\right) C \cdot \bullet \lambda^{t}\left(q^{-1}\right), \\
Z=M(q)^{2} \prod_{k>0}\left(1-Q q^{k}\right)^{k} .
\end{gathered}
$$

Where we have shifted $Q \rightarrow-Q$ in order to agree with the gluing rules of the topological vertex.

$T^{*}\left(\mathbf{P}^{1}\right) \times \mathbf{C}$ : This geometry can be obtained from local $\mathbf{P}^{1} \times \mathbf{P}^{1}$ in the limit when the area of one of the $\mathbf{P}^{1}$ 's is taken to infinity. It will be useful to discuss this example because, 


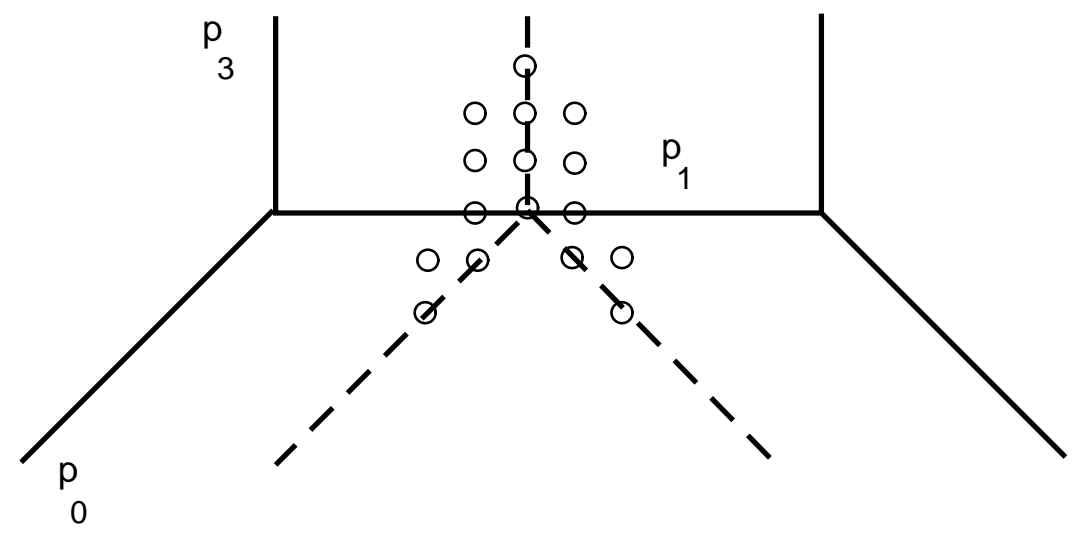

Fig. 23: Toric diagram of $T^{*}\left(\mathbf{P}^{1}\right) \times \mathbf{C}$. Dashed lines indicate the perpendicular slicing of the of the $3 d$ partitions leading to $2 d$ partitions on the dashed plane.

unlike the previous example, this is the simplest geometry that involves non-trivial framing. The linear sigma model charges for $T^{*}\left(\mathbf{P}^{1}\right)$ are given by $(1,1,-2)$, the field describing $\mathbf{C}$ is, however, uncharged under this $U(1)$,

$$
p_{1}+p_{2}-2 p_{0}=t, \quad p_{3} \geq 0
$$

fig. 23 shows the toric diagram of this geometry. As discussed previously, the partition function is given by

$$
Z=\sum_{\pi_{1}, \pi_{2}} q^{\left|\pi_{1}\right|+\left|\pi_{2}\right|+\Delta(\lambda) \mid} Q^{|\lambda|}=\sum_{\lambda} Q^{\lambda} \sum_{\pi_{1,2}} q^{\left|\pi_{1}\right|+\left|\pi_{2}\right|+\Delta(\lambda)} .
$$

Where the sum is over two $3 \mathrm{~d}$ partitions $\pi_{1}, \pi_{2}$ such that the $2 \mathrm{~d}$ partition obtained by slicing them in the direction of the $\mathbf{P}^{1}$ is $\lambda$, and $\Delta(\lambda)$ is the volume between the two perpendicular slices shown in fig. 23 . If the $2 \mathrm{~d}$ partition $\lambda$ is rotated on its first column by $\pi / 4$ the volume obtained is given by $\frac{1}{2} \sum_{i} \lambda_{i}^{2}=\frac{\|\lambda\|^{2}}{2}$. This was the case in the previous example. In the example we are considering now the rotation is such that it maps $(0,1)$ vector in the $\left(p_{1}, p_{0}\right)$ plane to $(2,1)$. Thus $\Delta(\lambda)=\sum_{i} \lambda_{i}^{2}=\|\lambda\|^{2}$. Using the definition of the topological vertex in terms of $3 \mathrm{~d}$ partitions [9] we get

$$
\begin{aligned}
Z & =M(q)^{2} \sum_{\lambda} Q^{\lambda} C_{\bullet \bullet \lambda}\left(q^{-1}\right) C \bullet \bullet \lambda^{t}\left(q^{-1}\right) q^{\Delta(\lambda)-\left(\|\lambda\|^{2}+\left\|\lambda^{t}\right\|^{2}\right) / 2} \\
& =M(q)^{2} \sum_{\lambda} Q^{\lambda} C_{\bullet \bullet \lambda}\left(q^{-1}\right) C \bullet \bullet \lambda^{t}\left(q^{-1}\right) q^{\left(\|\lambda\|\left\|^{2}-\right\| \lambda^{t} \|^{2}\right) / 2}
\end{aligned}
$$


Using the relation $\|\lambda\|^{2}-\left\|\lambda^{t}\right\|^{2}=\kappa(\lambda)=|\lambda|+\sum_{i} \lambda_{i}\left(\lambda_{i}-2 i\right)$ the partition function becomes

$$
\begin{gathered}
Z=M(q)^{2} \sum_{\lambda} Q^{|\lambda|} C_{\bullet \bullet \lambda}\left(q^{-1}\right) C_{\bullet \bullet \lambda^{t}}\left(q^{-1}\right) q^{\kappa(\lambda) / 2} \\
=M(q)^{2} \sum_{\lambda} Q^{|\lambda|} C_{\bullet \bullet \lambda}(q) C_{\bullet \bullet \lambda}(q) .
\end{gathered}
$$

Where in the last equation we used the property of the Schur function $s_{\lambda^{t}}\left(q^{-i+1 / 2}\right)=$ $s_{\lambda}\left(q^{-i+1 / 2}\right) q^{-\kappa(\lambda) / 2}$. Thus we see that correct framing factors follow from our prescription if points on the integral lattice are counted carefully.

\section{Target space theory viewpoint}

The aim of this section is to propose a precise (gauge) theory which plays the role of target space Kähler gravity. Just like the three (and two) dimensional gravities have gauge formulations (involving $S L_{2}, E(2)$, etc.), Kähler gravity in any dimension is in some sense a $U(1)$ gauge theory. In this section we first motivate the choice of the $U(1)$ gauge theory as a twisted version of the maximally supersymmetric theory in 6 dimensions. We then discuss how toric symmetry localizes the path-integral for such a theory on certain toric invariant subspaces. The objects of relevance in the gauge theory are $6 \mathrm{~d}$ instantons, which are a singular configuration, very much like the point-like $U(1)$ instantons in 4 dimensions. To make the point-like instantons more well defined目 we also study a certain non-commutative deformation of the $U(1)$ gauge theory, just as in the 4 dimensional case 29.

To motivate the following construction let us recapitulate what we have said so far. We want to study Kähler gravity on the space $X$. The space $X$ is a noncompact (in most of what follows) toric Calabi-Yau manifold. It comes with some base Calabi-Yau metric and the corresponding Kähler form $k_{0}$. We want to study all Kähler metrics which asymptotically approach $k_{0}$. We can write:

$$
k=k_{0}+g_{s} F
$$

3 It is also interesting to pursue the other possibilities of making the $U(1)$ instantons well defined, which came up in the matrix model approach to $\mathcal{N}=1$ supersymmetric gauge theory 27,28]: Either considering $U(N)$ theory and taking the $N \rightarrow 1$ limit, or more fundamentally replacing $U(1)$ by the supergroup $U(k+1 \mid k)$. 
where

$$
F^{2,0}=0, \quad d F=0
$$

Here the $(2,0)$ projection is performed in the fixed complex structure of $X$ (of course, we only need the almost complex structure, in agreement with the general philosophy of type A topological strings). The putative Kähler gravity equations of motion impose some constraints on $F^{1,1}$. In quantum theory we calculate a path integral over all possible $F$ 's, as well as the corresponding ghosts, auxiliary fields and so on, with some supermeasure, hopefully derived from the BV formalism of [4]. Apparently, this superintegral has a $Q$ symmetry, which according to the old Witten's argument localizes it onto the $Q$-fixed points, which are the special solutions of the Kähler gravity equations of motion.

Now note that the problem is reformulated in terms of the closed two-form, which is of $(1,1)$ type and in addition satisfies some extra equation. Imagine in addition that $F$ is quantized, i.e. its periods are $\mathbf{Z}$-valued. Then we are dealing with $U(1)$ gauge theory on $X$. We shall loosely call the $Q$-invariant solutions of this theory instantons.

\subsection{Supersymmetric Yang-Mills theory on six-manifold}

What gauge theory should we look at? If we embed the topological string into the superstring, as in [7] then the most natural choice is the maximally supersymmetric gauge theory, i.e. the reduction (with a twist) of the $\mathcal{N}=1$ super-Yang-Mills from ten to six dimensions. This theory was rediscovered in many forms in the context of topological gauge theories in [30,31,32]. In general one can study this theory for any gauge group $G$. The $Q$-fixed field configurations there obey:

$$
\begin{gathered}
F_{A}^{2,0}=\bar{\partial}_{A}^{\dagger} \varphi \\
F_{A}^{1,1} \wedge k_{0} \wedge k_{0}+[\varphi, \bar{\varphi}]=\ell k_{0} \wedge k_{0} \wedge k_{0},
\end{gathered}
$$

where $\ell$ is some constant, fixed by the magnetic flux of the gauge bundle. Here $\varphi$ is the adjoint-valued $(3,0)$ form, which is the twisted complex scalar of the super-Yang-Mills theory. The second complex scalar $\phi$ remains untwisted and is the analogue of the adjoint Higgs field of the four dimensional $\mathcal{N}=2$ theory. It must be covariantly constant:

$$
d_{A} \phi=0
$$

The solutions of the system (6.3) (6.4) ought to be isolated, the corresponding index being zero, but in practice this may not be so. 
The bosonic part of the Lagrangian of our gauge theory is:

$$
\begin{gathered}
L_{b}=\frac{1}{2} \operatorname{tr}\left(d_{A} \phi \wedge \star d_{A} \bar{\phi}+[\phi, \bar{\phi}]^{2}+F_{A} \wedge \star F_{A}+D_{A} \varphi \wedge \star D_{A} \bar{\varphi}\right) \\
+\frac{\vartheta}{3 !(2 \pi)^{3}} \operatorname{tr} F_{A} \wedge F_{A} \wedge F_{A}
\end{gathered}
$$

the last line being the usual six dimensional theta term. The fermionic content of the theory is the following: a one-form $\psi$, two-form $\chi^{2,0}, \chi^{0,2}$, three form $\psi^{3,0}, \psi^{0,3}$, and two scalars $\eta, \bar{\eta}$ - sixteen real components in total, as it should been. The fermionic part of our Lagrangian is:

$$
\begin{aligned}
L_{f} & =\operatorname{tr}\left(k_{0} \wedge \chi^{2,0}\left(\bar{\partial}_{A} \psi^{0,1}+\bar{\partial}_{A}^{\dagger} \psi^{0,3}\right)+\eta\left(g^{i \bar{j}} D_{i} \psi_{\bar{j}}^{0,1}+\left[\bar{\varphi}, \psi^{0,3}\right]\right)+c . c .\right) \\
& +\operatorname{tr}\left(\left(k_{0} \wedge\left[\chi^{2,0}, \chi^{0,2}\right]+[\eta, \bar{\eta}]\right) \phi+\left([\psi, \star \psi]+\left[\psi^{3,0}, \psi^{0,3}\right]\right) \bar{\phi}\right)
\end{aligned}
$$

By rewriting the action (6.6) in the form:

$$
\begin{gathered}
S=\int_{X} \frac{1}{2} \operatorname{tr}\left(d_{A} \phi \wedge \star d_{A} \bar{\phi}+[\phi, \bar{\phi}]^{2}+\left|F_{A}^{2,0}+\bar{\partial}_{A}^{\dagger} \varphi\right|^{2}+\left|F_{k_{0}}^{1,1}\right|^{2}\right)+ \\
\frac{1}{2} \operatorname{tr}\left(F_{A} \wedge F_{A} \wedge k_{0}+\frac{\vartheta}{3} F_{A} \wedge F_{A} \wedge F_{A}\right)
\end{gathered}
$$

we see that the solutions of (6.3)(6.4) provide the minima of the Yang-Mills action in the given topological class, with precisely (2.2) as the instanton action (up to an additive constant, equal to the volume of $X$ ) where we identify $\vartheta=i g_{s}$ (see below for a more precise map).

It is a standard exercise in the instanton calculus to work out the measure on the space of collective coordinates. Linearization of the equations (6.3) (6.4) together with the gauge fixing condition define some elliptic complex, and the measure is the ratio of the determinants of the corresponding elliptic operators. Due to supersymmetry the determinants almost cancel, leaving essentially the zero modes to be taken care of. The standard by now trick is to lift these zero modes by working with the nonvanishing vacuum expectation value of the Higgs field $\phi$ and also by turning on the so-called $\Omega$-background (see below). Before we plunge into details, let us discuss the zero modes.

The moduli space $\mathcal{M}$ of solutions to (6.3) (6.4) for complex $X$ is a complex variety as well. Its holomorphic tangent space at the point $(A, \varphi)$ is the space of solutions of 
the linearized equations (6.3) up to linearized complexified gauge transformations, i.e. the cohomology of the complex:

$$
\begin{gathered}
\mathcal{T}_{(A, \varphi)} \mathcal{M}=H^{1} \\
0 \longrightarrow \Omega_{A}^{0,0} \longrightarrow \Omega_{A}^{0,1} \oplus \Omega_{A}^{0,3} \longrightarrow \Omega_{A}^{0,2} \longrightarrow 0 \\
\eta \in \Omega_{A}^{0,0} \mapsto \bar{\partial}_{A} \eta \oplus[\varphi, \eta] \in \Omega_{A}^{0,1} \oplus \Omega_{A}^{0,3} \\
\delta \bar{A} \oplus \delta \varphi \in \Omega_{A}^{0,1} \oplus \Omega_{A}^{0,3} \mapsto \bar{\partial}_{A} \delta \bar{A}+\bar{\partial}_{A}^{\dagger} \delta \varphi \in \Omega_{A}^{0,2}
\end{gathered}
$$

In addition, on top of $\mathcal{M}$, one finds the so-called obstruction bundle $\mathcal{N}$, or the antighost bundle in the physical terms, which is spanned by the zero modes of the conjugate operator, or the $H^{2}$ of the complex above (which one finds by writing out the fermionic kinetic term for the theory $(2.2))$ :

$$
\partial_{A}^{\dagger} \chi^{2,0}+\partial_{A} \eta=0, \quad \partial_{A} \chi^{2,0}+[\bar{\varphi}, \eta]=0
$$

Integrating over the fermionic zero modes along $\mathcal{N}$ brings down the Pfaffian of the curvature of $\mathcal{N}$. The instanton contribution comes out to be:

$$
\int_{\mathcal{M}} \operatorname{Pfaff}\left(\mathcal{R}_{\mathcal{N}}\right)=\operatorname{Euler}(\mathcal{N})
$$

Note that this is not the Euler characteristics of $\mathcal{M}$, unlike the four dimensional maximally supersymmetric case [33], because $\mathcal{N}$ does not, in general, coincide with $\mathcal{T} \mathcal{M}$, although it has the same rank.

\subsection{Toric localization}

Now recall that the space $X$ has a torus $\mathbf{T}^{3}$ worth of isometries. Then one can deform the Lagrangian (2.2), $L \rightarrow L_{\Omega}$ by placing the theory in the so-called $\Omega$-background, which in practice changes the terms $\left\|d_{A} \phi\right\|^{2}+\|[\phi, \bar{\phi}]\|^{2}$ to

$$
\left\|d_{A} \phi-\iota_{\Omega} F\right\|^{2}+\left\|[\phi, \bar{\phi}]-L_{\bar{\Omega}} \phi+L_{\Omega} \bar{\phi}\right\|^{2}
$$

where $\Omega$ and $\bar{\Omega}$ are two commuting vector fields on $X$ generating isometries (we won't see any of $\bar{\Omega}$ in what follows). The path integral in gauge theory will now localize onto the 
solutions of 6.3 (6.4) which in addition are $\Omega$-invariant, or, in other terms, such that the infinitesimal rotation of $X$ can be compensated by the infinitesimal gauge transformation:

$$
d_{A} \phi=\iota_{\Omega} F_{A}
$$

The energy density $\operatorname{tr} F_{A} \star F_{A}$ of the $\Omega$-invariant solution wants to concentrate near the $\Omega$-fixed submanifolds in $X$. Typically, the six dimensional instanton wants to look like a small size four dimensional instanton fibered over a two-sphere, which connects two fixed points $f_{1}$ and $f_{2}$ (and $\varphi$ vanishes). This takes care of the $c h_{2} \sim \operatorname{tr} F_{A} \wedge F_{A}$ part of the topological charge. In addition, for nontrivial $c h_{3} \sim \operatorname{tr} F_{A}^{3}$ the energy density has sharp peaks at the fixed points $f$ themselves.

Let us label the point-like instanton configurations concentrated near a point $f$ by $\pi_{f}\left(\pi_{f}\right.$ will turn out to be a $3 \mathrm{~d}$ partition) and the instantons spread over the two-sphere connecting two fixed points $f_{1}$ and $f_{2}$ by $\lambda_{f_{1} f_{2}}$ (later on this will be a $2 \mathrm{~d}$ partition). If we look at the same configuration in the opposite direction, we shall label the corresponding instanton by $\lambda_{f_{1} f_{2}}^{t}=\lambda_{f_{2} f_{1}}$.

$$
\begin{aligned}
& \operatorname{tr}\left(F_{A} \wedge F_{A}\right) \sim \sum_{f_{1}, f_{2}}\left|\lambda_{f_{1}, f_{2}}\right| \delta_{\mathbf{P}_{f_{1} f_{2}}^{1}}^{(4)} \\
& \operatorname{tr}\left(F_{A} \wedge F_{A} \wedge F_{A}\right) \sim \sum_{f}\left|\pi_{f}\right| \delta_{f}^{(6)}
\end{aligned}
$$

Now let us scale the physical metric on $X$ by a large factor (using the complex nature of $k_{0}$ we can keep the class of $k_{0}$ intact), and apply field theory factorization (the fermionic symmetry preserved by $\Omega$-background guarantees that the partition function does not change). The path integral will therefore split as the integral over local $\mathbf{R}^{6}$ patches near each $f$, with asymptotic boundary conditions set by $\lambda_{f f^{\prime}}$ for all $f^{\prime}$ connected to $f$. In turn, the latter will be the sum over $\pi_{f}$ of the instanton contributions evaluated at $\pi_{f}$. Let us denote it by:

$$
\Gamma_{\lambda \mu \nu}(q, \Omega)=\int_{\text {asymptotics } \lambda \mu \nu} D A D \phi D \varphi D \psi D \chi D \eta e^{-\int_{\mathbf{R}^{6}} L_{\Omega}}
$$

where $q=e^{i \vartheta}$, and by asymptotics $\lambda \mu \nu$ we mean 4 :

$$
\begin{aligned}
& z_{1} \rightarrow \infty, z_{2}, z_{3} \text { finite }: A \rightarrow \lambda \text { instanton on } \mathbf{R}^{4} \\
& z_{2} \rightarrow \infty, z_{1}, z_{3} \text { finite }: A \rightarrow \mu \text { instanton on } \mathbf{R}^{4} \\
& z_{3} \rightarrow \infty, z_{2}, z_{1} \text { finite }: A \rightarrow \nu \text { instanton on } \mathbf{R}^{4}
\end{aligned}
$$

4 Recall that torus-invariant instantons in $\mathbf{R}^{4}$ are in one to one correspondence with $2 \mathrm{~d}$ Young diagrams with instanton charge being the number of boxes in the Young diagram [12]. 
Then the partition function on $X$ is given by:

$$
Z_{X}(q, \Omega)=\sum_{\lambda_{e}} \prod_{f} \Gamma_{\lambda_{e_{1}} \lambda_{e_{2}} \lambda_{e_{3}}}\left(q, \Omega_{f}\right) \prod_{e} e^{-t_{e}\left|\lambda_{e}\right|}
$$

Here $\Omega_{f}$ is the local expression for $\Omega$ near $f, e_{1}, e_{2}, e_{3}$ are the $\Omega$-invariant complex lines, passing through $f,\left|\lambda_{e}\right|$ is the four dimensional instanton action, and $t_{e}$ is the Kähler class of the line $e$. For local $\mathbf{P}^{1}$ the formula (6.17) specializes to:

$Z_{\text {local } \mathbf{P}^{1}}(q, \vec{\epsilon})=\sum_{\lambda} e^{-t|\lambda|} \Gamma_{\bullet, \bullet, \lambda}\left(q, \epsilon_{1}-\epsilon_{0}, \epsilon_{2}+\epsilon_{0}, \epsilon_{3}+\epsilon_{0}\right) \Gamma_{\bullet, \bullet, \lambda^{t}}\left(q, \epsilon_{0}-\epsilon_{1}, \epsilon_{2}+\epsilon_{1}, \epsilon_{3}+\epsilon_{1}\right)$

\subsection{Gauge vertex}

We now wish to learn more about the vertex $\Gamma_{\lambda \mu \nu}(q, \Omega)$. To this end we study more closely the gauge theory.

Flat space revisited: noncommutative interpretation: In the case $G=U(1)$ there are hardly any nontrivial solutions to (6.3) (6.4). There is, however, a simple way out of this, at least on $X=\mathbf{R}^{6}$ or its orbifolds. Replace $X$ by its noncommutative deformation. Say, on $\mathbf{R}^{6}$ we work with the algebra:

$$
\left[x^{m}, x^{n}\right]=i \theta^{m n}, \quad m, n=1, \ldots, 6
$$

where $\theta^{m n}$ is some constant anti-symmetric matrix, which we shall assume to be of maximal rank. Moreover, by an orthogonal rotation we can bring $\theta$ into the form:

$$
\theta=\left(\begin{array}{cccccc}
0 & \theta_{1} & & & & \\
-\theta_{1} & 0 & & & & \\
& & 0 & \theta_{2} & & \\
& & -\theta_{2} & 0 & & \\
& & & & 0 & \theta_{3} \\
& & & & -\theta_{3} & 0
\end{array}\right), \quad \theta_{\alpha}>0
$$

It is convenient to work with the fields:

$$
X^{n}=x^{n}+i \theta^{n m} A_{m}(x)
$$

as opposed to the gauge fields $A_{m}$. The field strength is related to the commutator:

$$
\left[X^{m}, X^{n}\right]-i \theta^{m n}=\theta^{m m^{\prime}} \theta^{n n^{\prime}} F_{m^{\prime} n^{\prime}}
$$


and the equations $(6.3)(6.4)$ are replaced by:

$$
\begin{aligned}
& {\left[Z^{\alpha}, Z^{\beta}\right]+\varepsilon^{\alpha \beta \gamma}\left[Z_{\gamma}^{\dagger}, \varphi\right]=0, \alpha, \beta=1,2,3} \\
& {\left[Z^{\alpha}, Z_{\alpha}^{\dagger}\right]+\left[\varphi, \varphi^{\dagger}\right]=3}
\end{aligned}
$$

where we have introduced

$$
Z^{1}=\frac{1}{\sqrt{2 \theta_{1}}}\left(X^{1}+i X^{2}\right), \quad \text { etc. }
$$

The equation $d_{A} \phi=0$ is replaced by $\left[Z^{\alpha}, \Phi\right]=0$.

In the vacuum:

$$
Z^{\alpha}=a_{\alpha}, \varphi=0, \Phi=\phi \cdot 1 \quad\left[a_{\alpha}, a_{\beta}^{\dagger}\right]=\delta_{\alpha \beta}
$$

Here is an example of the nontrivial solution to (6.23), found in [34] (for $m=1$ in [35]):

$$
Z^{\alpha}=S a_{\alpha}\left[1-\frac{m(m+1)(m+2)}{N(N+1)(N+2)}\right]^{\frac{1}{2}} S^{\dagger}
$$

where:

$$
N=a_{1}^{\dagger} a_{1}+a_{2}^{\dagger} a_{2}+a_{3}^{\dagger} a_{3}
$$

and $S$ is the so-called Murray-von Neumann partial isometry:

$$
S S^{\dagger}=1, \quad S^{\dagger} S=1-\sum_{i+j+k<m}|i, j, k\rangle\langle i, j, k|
$$

and we have used the standard oscillator representation of the algebra of $a_{\alpha}, a_{\beta}^{\dagger}$. This solution is related to the blow up of the point on $\mathbf{C}^{3}$ discussed above, with the Kähler form of exceptional $\mathbf{C P}^{2}$ being $m$ times the standard Fubini-Study form.

Toric localization of noncommutative gauge theory: Now we deform our gauge theory further, by turning on the so-called $\Omega$-background [12,36], which utilizes the toric symmetry of $\mathbf{R}^{6}$. The equation (6.5) gets deformed to:

$$
\left[\Phi, Z^{\alpha}\right]=\epsilon_{\alpha} Z^{\alpha}
$$

where $\epsilon_{1}, \epsilon_{2}, \epsilon_{3}$ are the parameters of the $\Omega$-background, the angles of the infinitesimal $\mathbf{T}^{3}$ rotation of $\mathbf{R}^{6}$. One can show that the solutions to $(6.23)(6.29)$ have the following form:

$$
Z^{\alpha}=S \Lambda_{\vec{n}}^{-\frac{1}{2}} a_{\alpha} \Lambda_{\vec{n}}^{\frac{1}{2}} S^{\dagger}, \quad \Phi=S\left(\epsilon_{1} a_{1}^{\dagger} a_{1}+\epsilon_{2} a_{2}^{\dagger} a_{2}+\epsilon_{3} a_{3}^{\dagger} a_{3}\right) S^{\dagger}
$$


and the partial isometry identifies the Hilbert space $\mathcal{H}$ of all states of the triple of harmonic oscillators,

$$
\mathcal{H}=\mathbf{C}\left[a_{1}^{\dagger}, a_{2}^{\dagger}, a_{3}^{\dagger}\right]|0,0,0\rangle
$$

with its subspace:

$$
\mathcal{H}_{\mathcal{I}}=\mathcal{I}\left(a_{1}^{\dagger}, a_{2}^{\dagger}, a_{3}^{\dagger}\right)|0,0,0\rangle
$$

where $\mathcal{I} \subset \mathbf{C}\left[w_{1}, w_{2}, w_{3}\right]$ is an ideal in the ring of polynomials, generated by monomials. In the example above the ideal in question is spanned by

$$
w_{1}^{i} w_{2}^{j} w_{3}^{k}, \quad i+j+k \geq m
$$

Any such monomial-generated ideal $\mathcal{I}$ defines the so-called three-dimensional partition (which is infinite for nontrivial asymptotics):

$$
\pi=\left\{(i, j, k) \mid i, j, k \geq 1, w_{1}^{i-1} w_{2}^{j-1} w_{3}^{k-1} \notin \quad \mathcal{I}\right\}
$$

The physical picture of the localization is the following: the instantons (which are pointlike, or fuzzy of the "size" $\sim \theta$ ) tend to sit on top of each other, near the origin in $\mathbf{R}^{6}$, the fixed point of the spatial rotation. They can also, as we discussed above, concentrate along the coordinate axes, which are also invariant under rotation, and asymptote to the ordinary four dimensional noncommutative instantons [37], invariant under rotations, and labelled by the two-dimensional partitions.

Back to determinants: What is the contribution of such an instanton? We should expand the super-Yang-Mills action and pick up the determinants of the bosonic and fermionic fluctuations. The ratio comes out to be (cf. [38]):

$$
e^{i \vartheta c h_{3}} \frac{\operatorname{Det}(\operatorname{ad} \Phi) \operatorname{Det}\left(\operatorname{ad} \Phi+\epsilon_{1}+\epsilon_{2}\right) \operatorname{Det}\left(\operatorname{ad} \Phi+\epsilon_{3}+\epsilon_{2}\right) \operatorname{Det}\left(\operatorname{ad} \Phi+\epsilon_{3}+\epsilon_{1}\right)}{\operatorname{Det}\left(\operatorname{ad} \Phi+\epsilon_{1}+\epsilon_{2}+\epsilon_{3}\right) \operatorname{Det}\left(\operatorname{ad} \Phi+\epsilon_{1}\right) \operatorname{Det}\left(\operatorname{ad} \Phi+\epsilon_{2}\right) \operatorname{Det}\left(\operatorname{ad} \Phi+\epsilon_{3}\right)}
$$

where ad $\Phi$ denotes the operator $[\Phi, \cdot]$, and one should omit the zero modes (actually these are absent).

Perhaps a more suggestive form of the ratio of the determinants is given by the proper time representation:

$$
Z_{\mathcal{I}}\left(\epsilon_{1}, \epsilon_{2}, \epsilon_{3}\right)=\exp i \vartheta\left[\operatorname{Tr}_{\mathcal{H}} e^{t \Phi}\right]_{t^{0}}+\int_{0}^{\infty} \frac{d t}{t}\left(1-e^{t \epsilon_{1}}\right)\left(1-e^{t \epsilon_{2}}\right)\left(1-e^{t \epsilon_{3}}\right) \operatorname{Tr}_{\mathcal{H}} e^{t \Phi} \operatorname{Tr}_{\mathcal{H}} e^{-t \Phi}
$$


At any rate, this is an explicit function of the ideal $\mathcal{I}$ and can be calculated. We can actually split it as a contribution of the vacuum solution with given asymptotics, and then some finite number of instantons on top of it. For the vacuum:

$$
\operatorname{Tr}_{\mathcal{H}} e^{t \Phi}=\frac{1}{\left(1-e^{t \epsilon_{1}}\right)\left(1-e^{t \epsilon_{2}}\right)\left(1-e^{t \epsilon_{3}}\right)}
$$

for ideal $\mathcal{I}$ corresponding to the partition $\pi$ (perhaps infinite):

$$
\operatorname{Tr}_{\mathcal{H}} e^{t \Phi}=\frac{1}{\left(1-e^{t \epsilon_{1}}\right)\left(1-e^{t \epsilon_{2}}\right)\left(1-e^{t \epsilon_{3}}\right)}-\sum_{(i, j, k) \in \pi} e^{t\left(\epsilon_{1}(i-1)+\epsilon_{2}(j-1)+\epsilon_{3}(k-1)\right)},
$$

and the vacuum with fixed asymptotics $\lambda_{1}, \lambda_{2}, \lambda_{3}$ :

$$
\operatorname{Tr}_{\mathcal{H}} e^{t \Phi}=\frac{1}{\left(1-e^{t \epsilon_{1}}\right)\left(1-e^{t \epsilon_{2}}\right)\left(1-e^{t \epsilon_{3}}\right)}-\left[\frac{R_{\lambda_{3}}\left(e^{t \epsilon_{1}}, e^{t \epsilon_{2}}\right)}{\left(1-e^{t \epsilon_{3}}\right)}+(3 \rightarrow 2 \rightarrow 1)\right]
$$

where

$$
R_{\lambda}(x, y)=\sum_{(i, j) \in \lambda} x^{i-1} y^{j-1}, \quad|\lambda|=R_{\lambda}(1,1)
$$

The instanton weight $(6.36)$ splits, as:

$$
Z_{\mathcal{I}}\left(\epsilon_{1}, \epsilon_{2}, \epsilon_{3}\right)=Z_{\lambda \mu \nu}^{v a c}\left(\epsilon_{1}, \epsilon_{2}, \epsilon_{3}\right) Z_{\mathcal{I}}^{f i n}\left(\epsilon_{1}, \epsilon_{2}, \epsilon_{3}\right)
$$

where the last factor is a product of the finite (and equal) number of terms in the numerator and the denominator. Finally, the most amazing property of the instanton weight (6.36) is that for $\epsilon_{1}+\epsilon_{2}+\epsilon_{3}=0$ the finite part $Z^{\text {fin }}$ is equal to $(-1)^{[\mathcal{I}]}$, with $[\mathcal{I}]$ defined more precisely below, which allows us to identify, $\left.\Gamma_{\lambda \mu \nu}\right|_{C Y} \sim C_{\lambda \mu \nu}$.

Remark. Similar localization of supersymmetric gauge theory (not the simplification of the $Z^{\text {fin }}$ factors) on toric manifolds holds in any number of dimensions. In particular, in four real dimensions one gets an expression for Donaldson invariants on any toric surface in terms of the instanton partition function on $\mathbf{R}^{4}$ [39]. In the case of $X=\widehat{\mathbf{C}^{2}}$, the blowup of a point on $\mathbf{C}^{2}$ this was essentially used in [40].

The formula (6.35) is written for $X=\mathbf{R}^{6}$. For general toric $X$ it should be modified. The factorization formula (6.17) suggests that the determinants of the fluctuations about the instantons localized near the fixed points in $X$ and the connecting them spheres should factorize as well. In fact, by relating the determinant ratio with the index of appropriate family of elliptic operators (like $\bar{\partial}_{A} \oplus \bar{\partial}_{A}^{\dagger}$ ), and using for the latter the equivariant AtiyahSinger index theorem, which expresses it as an integral over $X$, and then using the latter 
Duistermaat-Heckmann [41] formula we shall get precisely (6.17). Again, for the vector field $\Omega$ preserving the holomorphic $(3,0)$ form the determinants cancel up to a well-defined sign factor, discussed below.

Vertex and framing: Let us discuss a little bit more the vertex and the instanton factors. We wish to weigh the instantons with the factor:

$$
\exp -\left[\frac{1}{8 \pi^{2}} \int_{X} k_{0} \wedge \operatorname{tr} F_{A}^{2}+\frac{\vartheta}{48 \pi^{3}} \int_{X} \operatorname{tr} F_{A}^{3}\right]
$$

which we can calculate for the $\Omega$-invariant instantons using localization:

$$
\begin{aligned}
& -\frac{1}{8 \pi^{2}} \int_{X} k_{0} \wedge \operatorname{tr} F_{A}^{2}=\sum_{f} \frac{H_{f} \mathcal{E}_{f}^{(2)}}{\epsilon_{1 f} \epsilon_{2 f} \epsilon_{3 f}} \\
& \frac{i}{48 \pi^{3}} \int_{X} \operatorname{tr} F_{A}^{3}=\sum_{f} \frac{\mathcal{E}_{f}^{(3)}}{\epsilon_{1 f} \epsilon_{2 f} \epsilon_{3 f}}
\end{aligned}
$$

Here:

$$
\mathcal{E}_{f}(t)=1-\left(1-e^{t \epsilon_{1 f}}\right)\left(1-e^{t \epsilon_{2 f}}\right)\left(1-e^{t \epsilon_{3 f}}\right) \sum_{(i, j, k) \in \pi_{f}} e^{t\left(\epsilon_{1 f}(i-1)+\epsilon_{2 f}(j-1)+\epsilon_{3 f}(k-1)\right)},
$$

$\mathcal{E}_{f}^{(n)}$ denotes the coefficient in front of $t^{n}$ in the expansion of (6.43) near $t=0$, and $H_{f}$ is the value of the Hamiltonian (3.8) at the fixed point $f$ (note that shifting $H$ by constant won't affect (6.42) for compact $X$ ). Also, another application of the fixed point formula gives:

$$
t_{e}=\frac{H_{f_{1}}-H_{f_{2}}}{\epsilon_{e}}
$$

for $e=\left(f_{1}, f_{2}\right)$. The character $\mathcal{E}_{f}$ also appears in the expression for the ratio of the determinants:

$$
Z_{\mathcal{I}}(\vec{\epsilon})=\exp \int^{\infty} \frac{d t}{t} \sum_{f} \frac{\mathcal{E}_{f}(t) \mathcal{E}_{f}(-t)}{\left(1-e^{-t \epsilon_{1 f}}\right)\left(1-e^{-t \epsilon_{2 f}}\right)\left(1-e^{-t \epsilon_{3 f}}\right)}
$$

We now want to specialize our torus action to respect the Calabi-Yau condition. Now, by examining (6.42) (6.43) we see that the $c h_{2}$ part of the instanton action, when summed over $f$ 's will reduce to

$$
-\sum_{e} t_{e}\left|\lambda_{e}\right|
$$


where we should use (6.44). The $c h_{3}$ part actually depends on one parameter, which we can take to be $x=\epsilon_{2 f} / \epsilon_{1 f}$. The local contribution to the $c h_{3}$ part of the instanton action is equal to:

$$
\begin{aligned}
& \frac{\mathcal{E}_{f}^{(3)}}{\epsilon_{1 f} \epsilon_{2 f} \epsilon_{3 f}}=\left|\pi_{f}\right|+I_{\lambda \mu \nu} {\left[\sum_{(i, j) \in \lambda}\left(i-\frac{1}{2}\right) x+\left(j-\frac{1}{2}\right)(-1-x)\right]+} \\
& I_{\lambda \mu \nu}= {\left[\frac{1}{x} \sum_{(j, k) \in \mu}\left(j-\frac{1}{2}\right)(-1-x)+\left(k-\frac{1}{2}\right)\right]+} \\
& {\left[-\frac{1}{1+x} \sum_{(k, i) \in \nu}\left(k-\frac{1}{2}\right)+\left(j-\frac{1}{2}\right) x\right] }
\end{aligned}
$$

and

$$
\left|\pi_{f}\right|=\left[\sum_{(i, j, k) \in \pi_{f}, i, j, k \leq N} 1\right]-(N+1)(|\lambda|+|\mu|+|\nu|), \quad N \gg 0
$$

Local considerations do not give a canonical value for $x$. This is related to the framing of the topological vertex. However, in summing over the edges the $x$ dependence of the instanton action essentially cancels. More precisely, each edge $e=\left(f_{1}, f_{2}\right)$ comes with the weights $\left(m_{1 e}, m_{2 e}\right)$ 目 of the $\Omega$ action on the normal bundle to the corresponding sphere $\mathbf{P}_{e}^{1}$. The local weights $\left(\epsilon_{1 f}, \epsilon_{2 f}, \epsilon_{3 f}\right)$ at two poles of the sphere are related by:

$$
\left(\epsilon_{1 f_{1}}, \epsilon_{2 f_{1}}, \epsilon_{3 f_{1}}\right) \mapsto\left(\epsilon_{1 f_{1}}+m_{1 e} e_{3 f_{1}}, \epsilon_{2 f_{1}}+m_{2 e} \epsilon_{3 f_{1}},-\epsilon_{3 f_{1}}\right)=\left(\epsilon_{1 f_{2}}, \epsilon_{2 f_{2}}, \epsilon_{3 f_{2}}\right)
$$

as in the example of the non-generic sphere above. Thus the sum of the asymptotic contributions $I_{\lambda \mu \nu}$ collapses to:

$$
\sum_{e} \sum_{(i, j) \in \lambda_{e}}\left[m_{1 e}(i-1)+m_{2 e}(j-1)+1\right]
$$

As we said above the fluctuation determinants cancel up to a well-defined sign, which can be determined by slightly involved calculation:

$$
(-1)^{[\mathcal{I}]}, \quad[\mathcal{I}]=\sum_{f}\left|\pi_{f}\right|+\sum_{e} \sum_{(i, j) \in \lambda_{e}}\left(m_{1 e}(i-1)+m_{2 e}(j-1)+1\right)+m_{1 e}\left|\lambda_{e}\right| \bmod 2
$$

5 the order $\left(m_{1}, m_{2}\right)$ is correlated with the orientation of the edge, by the complex three-form on $\mathrm{CY}$ 
(recall that $m_{1}+m_{2}=2$ so mod 2 it does not matter which one of $m$ 's use). So all we are left with is the instanton weight (6.41). The vertex factor simply reduces to summation over all $\pi_{f}$ with the asymptotics $\lambda \mu \nu$ with the weight

$$
(-1)^{I} e^{i \vartheta I}=(-q)^{I}
$$

where

$$
I=\sum_{f}\left|\pi_{f}\right|+\sum_{e} \sum_{(i, j) \in \lambda_{e}}\left[m_{1 e}(i-1)+m_{2 e}(j-1)+1\right]
$$

Combining this with the minus sign on the edges, as in (6.51), we conclude:

$$
Z_{X}(q, t)=\sum_{\left\{\pi_{f}\right\}}(-q)^{I} \prod_{e}(-1)^{m_{e}\left|\lambda_{e}\right|} e^{-t_{e}\left|\lambda_{e}\right|}
$$

which reproduces the partition function for crystal melting with fixed asymptotes, including the framing factors (the edge signs), provided we identify:

$$
e^{-g_{s}}=-e^{i \vartheta}
$$

Thus, we have established the complete equivalence of the topological vertex counting of the all-genus string partition function, with the "U(1) maximally supersymmetric topologically twisted gauge theory" partition function on toric Calabi-Yau manifolds.

\section{Torsion free sheaves and general Calabi-Yau's}

So, on $\mathbf{C}^{3}$ we have a well-defined noncommutative gauge theory problem, and its solution can be phrased in purely commutative terms (for the holomorphic functions form a commutative subalgebra), i.e. in terms of ideals. What will replace this structure on a general manifold $X$, not necessarily toric? The global object that corresponds locally to an ideal is called an ideal sheaf. In each coordinate patch it is given by an ideal $\mathcal{I}_{U_{\alpha}}$ in the algebra of holomorphic functions $\mathcal{O}_{U_{\alpha}}$ on the patch, subject to the obvious consistency relations. It can be also characterized abstractly as rank one torsion free sheafs with trivial $c_{1}$. Here $c_{1}$ is the first Chern class of the ideal sheaf defined using any locally free resolution. Similarly, one defines $c_{2}$ and $c_{3}$, which need not be trivial. 
Any ideal sheaf $\mathcal{I}$ can be blown up. Blowup is a local construction and locally it can be described as follows. Suppose the ideal $I$ is generated by the polynomials $f_{1}(z), \ldots, f_{k}(z)$. Then the blowup $\hat{X}$ of $I$ is the closure in $X \times \mathbf{P}^{k-1}$ of the graph:

$$
\widehat{X}=\overline{\left\{\left(z,\left(f_{1}(z): \ldots: f_{k}(z)\right)\right) \mid z \in X \backslash Z\right\}}
$$

where $Z$ is the set of common zeroes of $f_{1}, \ldots, f_{k}$. For toric ideals, the generators $f_{1}, \ldots, f_{k}$ are in one-to-one correspondence with the inner corners of the 3d Young diagram, and the resulting space $\hat{X}$ has as its toric base $\Delta(\hat{X})$ the convex hull of the set of these corners. Observe that, by construction, the blowup comes with an embedding into a projective space and, hence, with a natural line bundle, Kähler metric, etc.

There is a natural moduli space of ideal sheaves $\mathcal{M}^{\text {sheaf }}$ on $X$, which is nothing but the Hilbert scheme of curves in $X$. This space is highly singular and the naive integration of the top Chern class of the obstruction bundle over it is ill-defined. However, the philosophy of virtual fundamental classes is applicable and yields a well-defined canonical zero-dimensional homology cycle [31] 42].

In the case of toric $X$ one can apply equivariant localization applied to a suitable class to verify that the sheaf cohomology produce the same result as the topological vertex [42], in accord with the gauge theory construction described in the previous section. This involves the identification of the "Donaldson-Thomas class" [31] as the relevant object to integrate over the moduli of ideal sheaves. It would be very interesting to relate more explicitly this class with that predicted by our gauge theory.

One can also extend the theory to more general $X$, first of all to compact CalabiYau's where there is no, up to date, effective technique of calculating the integrals over the virtual fundamental cycle of $\mathcal{M}^{\text {sheaf }}$, except for the trivial $c_{2}$ (maps to the point).

The gauge theory construction, however, provides hope for computational progress in the case of compact Calabi-Yau's, say quintic. The ground for this hope is the analogy with the four dimensional case, notably the Seiberg-Witten solution of the $\mathcal{N}=2$ theory [43], more precisely its recent formulation in terms of equivariant intersection theory on the moduli space of instantons on $\mathbf{R}^{4}[12]$. This is the analogue of our vertex. So we have now, at our disposal, an analogue of Seiberg-Witten ansatz (together with all gravitational couplings, necessary for putting the theory on a curved background). What is lacking, if anything, is the analogue of the full Seiberg-Witten geometry, including the monopole equations. This would be a fascinating subject to further develop. 
To support this analogy further, and explain what sort of objects we expect to see in our dual theory, let us recall the behavior of the maximally supersymmetric Yang-Mills theory in four dimensions. When appropriately twisted, this theory calculates the Euler characteristics of the appropriately completed and compactified instanton moduli spaces on the spacetime manifold $X$ [33]. The partition function, as a function of the complexified theta angle is a quasi-modular form, in accordance with S-duality [33]. For example, for $U(1)$ theory on $\mathrm{K} 3$, it is given by:

$$
Z_{K 3}(q, t)=\frac{\vartheta_{\Gamma^{3,19}}(t, \tau, \bar{\tau})}{\eta^{24}(q)}
$$

where $t \in H^{2}(K 3, \mathbf{R}), q=\exp 2 \pi i \tau$. The denominator, which comes from the point-like instantons, is the analogue of the MacMahon function to the power of $\chi / 2$ which is the expected result for topological strings on Calabi-Yau threefold.

\section{Acknowledgements.}

We would like to thank the first Simons Workshop on Mathematics and Physics where this work was initiated. We would also like to thank S. Gukov, K. Hori, G. Horowitz, S. Katz, H. Ooguri and A. Strominger for valuable discussions. NN is grateful to the High Energy Group at Harvard University and to the Physics Department of Rockefeller University for hospitality.

The research of AI and CV is supported in part by NSF grant DMS-0074329. CV is additionally supported by NSF grant PHY-9802709. The research of NN is partly supported by $\mathrm{P} \Phi \Phi И$ grant 03-02-17554 and by the grant HШ-1999.2003.2 for scientific

schools. A. O. was partially supported by DMS-0096246 and fellowships from the Packard foundation. 


\section{References}

[1] J. A. Wheeler, in "Relativity, Groups and Topology", edited by B. S. and C. M. DeWitt (Gordon and Breach, New York, 1964).

[2] S. W. Hawking, "Space-time Foam", Nucl. Phys. B144 (1978) 349-362.

[3] E. Witten, "Two-dimensional gravity and intersection theory on moduli space", Surveys Diff. Geom. 1 (1991) 243-310.

[4] M. Bershadsky, V. Sadov, "Theory of Kähler Gravity", Int. J. Mod. Phys. A11 (1996) 4689-4730, hep-th/9410011.

[5] R. Gopakumar, C. Vafa, "On the Gauge Theory/Geometry Correspondence", Adv. Theor. Math. Phys. 3 (1999) 1415-1443, hep-th/9811131

[6] N. Berkovits, H. Ooguri, C. Vafa, "On the Worldsheet Derivation of Large N Dualities for the Superstring", hep-th/0310118.

[7] M. Bershadsky, S. Cecotti, H. Ooguri, C. Vafa, "Kodaira-Spencer Theory of Gravity and Exact Results for Quantum String Amplitudes", Commun. Math. Phys. 165 (1994) 311-428, hep-th/9309140.

[8] I. Antoniadis, E. Gava, K. S. Narain, T. R. Taylor, "Topological Amplitudes in String Theory", Nucl. Phys. B413 (1994) 162-184, hep-th/9307158.

[9] A. Okounkov, N. Reshetikhin, C. Vafa, "Quantum Calabi-Yau and Classical Crystals", hep-th/0309208.

[10] H. Ooguri, A. Strominger, C. Vafa, work in progress.

[11] E. Witten, "Topology changing amplitudes in (2+1)-dimensional gravity", Nucl. Phys. B323 113, 1989.

[12] N. Nekrasov, "Seiberg-Witten prepotential via instanton counting", hep-th/0206161.

[13] E. Witten, "Chern-Simons Gauge Theory as a String Theory", Prog. Math. 133 (1995) 637-678, hep-th/9207094.

[14] M. Aganagic, A. Klemm, M. Marino, C. Vafa, "The topological vertex", hepth/0305132.

[15] T. Thiemann, "Lectures on loop quantum gravity," Lect. Notes Phys. 631 (2003) 41-135, gr-qc/0210094.

[16] H. W. Braden, N. Nekrasov, "Space-Time Foam From Non-Commutative Instantons", hep-th/9912019.

[17] S. Gukov, "Three-dimensional quantum gravity, Chern-Simons theory and the Apolynomial," hep-th/0306165.

[18] E. Witten, "Phases of $\mathrm{N}=2$ theories in two dimensions", Nucl. Phys. B403 (1993) 159-222, hep-th/9301042.

[19] M. Aganagic, C. Vafa, "Mirror symmetry, D-branes and counting holomorphic disks", hep-th/0012041. 
[20] A. Hanany, K. Hori, "Branes and $N=2$ theories in two dimensions", Nucl. Phys. B513 (1998) 119-174, hep-th/9707192.

[21] K. Hori, C. Vafa, "Mirror symmetry", hep-th/0002222.

[22] S. Gukov, C. Vafa, unpublished.

[23] R. Cerf, R. Kenyon, "The low-temperature expansion of the Wulff crystal in the 3D Ising model", Comm. Math. Phys. 222 (2001), no. 1, 147-179;

A. Okounkov, N. Reshetikhin, Journal of the American Mathematical Society, 16, no. 3 (2003), pp. 581-603.

[24] K. Hori, A. Iqbal, C. Vafa, "Mirror symmetry and D-branes", hep-th/0005247.

[25] H. Ooguri, C. Vafa, "Knot invariants and topological strings", Nucl. Phys. B577 (2000) 419-438, hep-th/991213.

[26] M. Aganagic, R. Dijkgraaf, A. Klemm, M. Marino, C. Vafa, "Topological strings and integrable hierarchies," to appear.

[27] R. Dijkgraaf, C. Vafa, "N=1 supersymmetry, deconstruction, and bosonic gauge theories," hep-th/0302011.

[28] M. Aganagic, K. Intriligator, C. Vafa, N.P. Warner, "The Glueball Superpotential," hep-th/0304271.

[29] N. Nekrasov, "Noncommutative Instantons Revisited", Comm. Math. Phys. 241 (2003) 143-160, hep-th/0010017.

[30] L. Baulieu, H. Kanno, and I. Singer, "Special quantum field theories in eight and other dimensions", hep-th/9704167;

B. Acharya, M. O'Loughlin, B. Spence, "Higher dimensional analogues of DonaldsonWitten theory", hep-th/9705138;

M. Blau, G. Thompson, "Euclidean SYM theories by time reduction and special holonomy manifolds," Phys. Lett. B415 (1997) 242-252, hep-th/9706225.

[31] S. Donaldson and R. Thomas, "Gauge theory in higher dimensions", in The geometric universe: science, geometry, and the work of Roger Penrose, S. Huggett et. al eds., Oxford Univ. Press, 1998.

[32] M. Bershadsky, V. Sadov, C. Vafa, "D-branes and topological field theories," Nucl. Phys. B463, (1996) 420, hep-th/9511222.

[33] C. Vafa, E. Witten, "A Strong Coupling Test of S-Duality", Nucl.Phys. B431 (1994) 3-77, hep-th/9408074

[34] P. Kraus, M. Shigemori, "Noncommutative instantons and the Seiberg-Witten map," hep-th/0110035.

[35] N. Nekrasov, "Lectures on open strings", hep-th/0203109.

[36] N. Nekrasov, A. Okounkov, "Seiberg-Witten theory and random partitions", hepth/0306238.

[37] N. Nekrasov, A. S. Schwarz, "Instantons on noncommutative $R^{4}$ and $(2,0)$ superconformal six-dimensional theory," Comm. Math. Phys. 198 (1998) 689, hep-th/9802068. 
[38] G. Moore, N. Nekrasov, S. Shatashvili, "Integrating over the Higgs branches", hepth/9712241;

G. Moore, N. Nekrasov, S. Shatashvili, "D-particle bound states and generalized instantons", hep-th/9803265.

[39] N. Nekrasov, "Localizing gauge theory", lecture notes, to appear.

[40] H. Nakajima, K. Yoshioka, "Instanton counting on blowup, I", math.AG/0306198.

[41] J. J. Duistermaat, G.J. Heckman, Invent. Math. 69 (1982) 259;

M. Atiyah, R. Bott, Topology 23 No 1 (1984) 1

[42] D. Maulik, N. Nekrasov, A. Okounkov, R. Pandharipande, "Gromov-Witten theory and Donaldson-Thomas theory", math.AG/0312059.

[43] N. Seiberg, E. Witten, "String theory and noncommutative geometry," JHEP 9909(1999) 032, , hep-th/9908142. 